\title{
HERSCHEL's “COLD DEBRIS DISKS”: BACKGROUND GALAXIES OR QUIESCENT RIMS OF PLANETARY SYSTEMS?
}

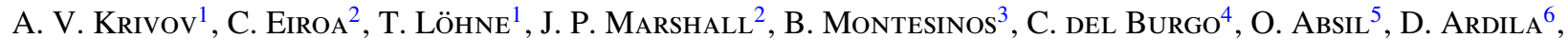 \\ J.-C. Augereau ${ }^{7}$, A. Bayo ${ }^{8,18}$, G. Bryden ${ }^{9}$, W. Danchi ${ }^{10}$, S. Ertel ${ }^{7}$, J. Lebreton ${ }^{7}$, R. Liseau ${ }^{11}$, A. Mora ${ }^{12}$, A. J. Mustill ${ }^{2}$, \\ H. Mutschke ${ }^{1}$, R. Neuhäuser ${ }^{1}$, G. L. Pilbratt ${ }^{13}$, A. Roberge ${ }^{10}$, T. O. B. Schmidt ${ }^{1}$, K. R. Stapelfeldt ${ }^{10}$, \\ Ph. Thébault ${ }^{14}$, Ch. Vitense ${ }^{1}$, G. J. White ${ }^{15,16}$, and S. Wolf ${ }^{17}$ \\ ${ }^{1}$ Astrophysikalisches Institut und Universitätssternwarte, Friedrich-Schiller-Universität Jena, Schillergäßchen 2-3, \\ D-07745 Jena, Germany; krivov@astro.uni-jena.de \\ 2 Departamento de Física Teórica, Facultad de Ciencias, Universidad Autónoma de Madrid, Cantoblanco, E-28049 Madrid, Spain \\ ${ }^{3}$ Departamento de Astrofísica, Centro de Astrobiología (CAB, CSIC-INTA), ESAC Campus, P.O. Box 78, E-28691 Villanueva de la Cañada, Madrid, Spain \\ ${ }^{4}$ Instituto Nacional de Astrofísica, Optica y Electrónica (INAOE), Apartado Postal 51 y 216, 72000 Puebla, Pue., Mexico \\ ${ }^{5}$ Institut d'Astrophysique et de Géophysique, Université de Liège, Allée du 6 Août 17, B-4000 Liège, Belgium \\ ${ }^{6}$ NASA Herschel Science Center, California Institute of Technology, 1200 East California Boulevard, Pasadena, CA 91125, USA \\ ${ }^{7}$ UJF-Grenoble 1/CNRS-INSU, Institut de Planétologie et d'Astrophysique de Grenoble (IPAG), UMR 5274, F-38041 Grenoble, France \\ ${ }^{8}$ European Southern Observatory, Alonso de Córdova 3107, Vitacura, Santiago, Chile \\ 9 Jet Propulsion Laboratory, California Institute of Technology, Pasadena, CA 91109, USA \\ ${ }^{10}$ NASA Goddard Space Flight Center, Exoplanets and Stellar Astrophysics, Code 667, Greenbelt, MD 20771, USA \\ ${ }^{11}$ Department of Earth and Space Sciences, Chalmers University of Technology, Onsala Space Observatory, SE-43992, Onsala, Sweden \\ ${ }^{12}$ ESA-ESAC Gaia SOC, P.O. Box 78, E-28691 Villanueva de la Cañada, Madrid, Spain \\ ${ }^{13}$ ESA Astrophysics \& Fundamental Physics Missions Division, ESTEC/SRE-SA, Keplerlaan 1, 2201 AZ Noordwijk, The Netherlands \\ ${ }^{14}$ LESIA, Observatoire de Paris, F-92195 Meudon Principal Cedex, France \\ 15 Department of Physics and Astrophysics, Open University, Walton Hall, Milton Keynes MK7 6AA, UK \\ ${ }^{16}$ Rutherford Appleton Laboratory, Chilton OX11 0QX, UK \\ ${ }^{17}$ Christian-Albrechts-Universität zu Kiel, Institut für Theoretische Physik und Astrophysik, Leibnizstr. 15, D-24098 Kiel, Germany \\ Received 2012 December 4; accepted 2013 May 23; published 2013 July 2
}

\begin{abstract}
Infrared excesses associated with debris disk host stars detected so far peak at wavelengths around $\sim 100 \mu \mathrm{m}$ or shorter. However, 6 out of 31 excess sources studied in the Herschel Open Time Key Programme, DUNES, have been seen to show significant—and in some cases extended—excess emission at $160 \mu \mathrm{m}$, which is larger than the $100 \mu \mathrm{m}$ excess. This excess emission has been attributed to circumstellar dust and has been suggested to stem from debris disks colder than those known previously. Since the excess emission of the cold disk candidates is extremely weak, challenging even the unrivaled sensitivity of Herschel, it is prudent to carefully consider whether some or even all of them may represent unrelated galactic or extragalactic emission, or even instrumental noise. We re-address these issues using several distinct methods and conclude that it is highly unlikely that none of the candidates represents a true circumstellar disk. For true disks, both the dust temperatures inferred from the spectral energy distributions and the disk radii estimated from the images suggest that the dust is nearly as cold as a blackbody. This requires the grains to be larger than $\sim 100 \mu \mathrm{m}$, even if they are rich in ices or are composed of any other material with a low absorption in the visible. The dearth of small grains is puzzling, since collisional models of debris disks predict that grains of all sizes down to several times the radiation pressure blowout limit should be present. We explore several conceivable scenarios: transport-dominated disks, disks of low dynamical excitation, and disks of unstirred primordial macroscopic grains. Our qualitative analysis and collisional simulations rule out the first two of these scenarios, but show the feasibility of the third one. We show that such disks can indeed survive for gigayears, largely preserving the primordial size distribution. They should be composed of macroscopic solids larger than millimeters, but smaller than a few kilometers in size. If larger planetesimals were present, then they would stir the disk, triggering a collisional cascade and thus causing production of small debris, which is not seen. Thus, planetesimal formation, at least in the outer regions of the systems, has stopped before "cometary" or "asteroidal" sizes were reached.
\end{abstract}

Key words: circumstellar matter - galaxies: statistics - planets and satellites: formation - protoplanetary disks stars: individual (HIP 29271, HIP 49908, HIP 109378, HIP 92043, HIP 171, HIP 73100)

Online-only material: color figures

\section{INTRODUCTION}

A significant fraction of main-sequence stars are found to be surrounded by detectable belts of debris composed of planetesimals and their dust (see, e.g., Wyatt 2008; Krivov 2010, for recent reviews). This material, together with planets, must represent the natural remnants of the systems' evolution during

18 Also at Max Planck Institut für Astronomie, Königstuhl 17, D-69117 Heidelberg, Germany. the protoplanetary phase. The observed debris disks typically reside on the outskirts of their host systems, just as for the solar system's Kuiper belt. The vast majority of these disks have been detected by observation of the thermal emission from their constituent dust at far-infrared (IR) wavelengths at levels above those predicted for the stellar photospheres. Several tens of these systems have been resolved (in at least one axis) providing crucial measurement of the dust spatial location around their host stars, though the bulk remain unresolved and can only be interpreted through their point-like thermal emission. 
One of the serendipitous results of the Herschel ${ }^{19}$ Open Time Key Programme (OTKP) DUNES (Eiroa et al. 2010) has been a tentative identification of a new class of "cold" debris disks (Eiroa et al. 2011). These are the cases where sources show significant IR excess at $160 \mu \mathrm{m}$ and possibly also at longer wavelengths, but smaller or no IR excess at $100 \mu \mathrm{m}$. This contrasts with all of the debris disks observed previously, whose thermal emission peaks at wavelengths $\lesssim 100 \mu \mathrm{m}$. Since the excess emission of the cold disk candidates is extremely weak, significant effort has gone into assessing the possible contamination by galactic or extragalactic emission, or even instrumental noise, and to show that the excess emission is likely to originate from the circumstellar dust.

Another Herschel OTKP, H-ATLAS, has reported two debris disk candidates with similar properties (Thompson et al. 2010). Their identification was based on the excess detection at $250 \mu \mathrm{m}$ and $350 \mu \mathrm{m}$ and upper limits at shorter and longer wavelengths. Rough estimates show that these may have properties similar to the DUNES cold disk candidates, except for substantially higher dust masses. Unfortunately, both candidates are located at large distances of $\sim 100-200 \mathrm{pc}$. As a result, the upper limits on the excess fluxes at $100 \mu \mathrm{m}$ and $160 \mu \mathrm{m}$ are very weak, as are the upper limits on the disk radii. This renders the formation of any definite conclusions extremely difficult, so that the H-ATLAS candidates are not further discussed here.

Assuming that the DUNES "cold disks" are true disks, the inferred dust temperatures are as low as $T_{d} \sim 20-30 \mathrm{~K}$ (Eiroa et al. 2011). Even though these disks have low fractional luminosities $\left(f_{d} \sim 10^{-6}\right)$, some of them reveal extended emission, allowing one to roughly estimate how far from the star the dust is located. The dust temperatures derived from the spectral energy distributions (SEDs) turn out to be comparable to the blackbody temperatures at the dust location. For those sources with point-like emission, we can place an upper limit on the disk radius, constraining the maximum distance from the star, where dust is still allowed to be for the disk to remain unresolved. In these cases too, the observed dust emission is nearly as cold as the blackbody emission at that distance.

Explaining how the material in true cold disks can be as cold as inferred is a challenge. The nearly blackbody temperatures of grains are suggestive of large grains (compared to far-IR wavelengths). It is not clear whether the requirement of large sizes can be relaxed by assuming dust compositions with a low absorption in the visible, such as icy grains. The lack of small grains would be difficult to explain because interpretations of multiwavelength resolved images of numerous debris disks performed so far reveal dominating sizes to lie in the micrometer range (see, e.g., Wyatt 2008; Krivov 2010 and references therein). This is also in accord with collisional models of debris disks that robustly predict all of the grains down to several times the radiation pressure blowout limit to be present (e.g., Wyatt et al. 1999; Wyatt \& Dent 2002; Krivov et al. 2006; Thébault \& Augereau 2007). Note that all of the DUNES targets are solartype (FGK) stars, for which the blowout radius - the radius of the smallest grains that can stay in bound orbits around the star against the radiation pressure-is $\lesssim 1 \mu \mathrm{m}$.

This paper extends the previous analysis (Eiroa et al. 2011) from three to all six cold disk candidates identified by DUNES; revisits the hypotheses of unrelated sources and "false alarms"; and, assuming that one or more of the candidates are real disks,

\footnotetext{
19 Herschel is an ESA space observatory with science instruments provided by European-led Principal Investigator consortia and with important participation from NASA.
}

explores possible reasons for the cold disk phenomenon. In Section 2, we describe a selection of cold disk candidates and re-address the disambiguation issue with possible background galaxies. In Section 3, we constrain the properties of the emitting material, trying to find grain sizes and compositions that are compatible with the observational data. In Section 4, we attempt to find an appropriate physical regime for debris disks that would provide the grains with the properties found in Section 3. Section 5 contains our conclusions and a discussion, and Section 6 offers a short summary of our findings.

\section{COLD DISK CANDIDATES}

\subsection{Characterization of Candidates}

The OTKP DUNES (Eiroa et al. 2010) ${ }^{20}$ is a survey of nearby ( $d \lesssim 20-25 \mathrm{pc}$ ), Sun-like (FGK) stars that used Herschel (Pilbratt et al. 2010) PACS (Poglitsch et al. 2010) and SPIRE (Griffin et al. 2010) scan map observations in six broad photometric bands around 70, 100, 160, 250, 350, and $500 \mu \mathrm{m}$. The DUNES strategy has been to integrate as deeply as needed to reach the photospheric level (at $100 \mu \mathrm{m}$ ) of all of the sources, facilitating detection of faint excess emission. In the entire DUNES data set of 133 stars, we have identified 31 sources as having a significant excess at least at one of these wavelengths (Eiroa et al. 2013). Of these 31, 6 have been classified as cold disk candidates, showing a significant (at least $>3 \sigma$ ) excess at $160 \mu \mathrm{m}$ that exceeds the excess at $100 \mu \mathrm{m}$ (if the latter is present at all). Three objects (HIP 29271, HIP 49908, and HIP 109378) have been discussed in more detail in Eiroa et al. (2011). The three additional candidates are HIP 171, HIP 73100, and HIP 92043.

The observations, data reduction procedures, photometry calculations, and the method of computing the expected photospheric fluxes at PACS and SPIRE wavelengths for all sources in the DUNES sample are described in detail in Eiroa et al. (2013). ObsIDs for the PACS and SPIRE observations are also listed there. Nonetheless, for the reader's convenience, here we give some details of the Herschel observations, data reduction, photometric estimates, and photospheric predictions specific to the six stars in question.

PACS scan map observations consisted of 10 legs of $3^{\prime}$ length, with a $4^{\prime \prime}$ separation between legs, scanning at the medium slew speed $\left(20^{\prime \prime} \mathrm{s}^{-1}\right)$. Each target was observed at two array orientation angles $\left(70^{\circ}\right.$ and $\left.110^{\circ}\right)$ to improve noise suppression and to assist in the removal of low-frequency $(1 / f)$ noise, instrumental artifacts, and glitches from the images. The SPIRE observation of HIP 92043 consisted of five repeats of the small scan map mode, ${ }^{21}$ producing a fully sampled map covering a region $4^{\prime}$ around the target. PACS and SPIRE observations were reduced using the Herschel Interactive Processing Environment (HIPE; Ott 2010) user release version 7.2, PACS calibration version 32, and SPIRE calibration version 8.1. The individual PACS scans were processed with a high-pass filter to remove background structure, using high-pass filter radii of 15 frames at $70 \mu \mathrm{m}, 20$ frames at $100 \mu \mathrm{m}$, and 25 frames at $160 \mu \mathrm{m}$, suppressing structure larger than $62^{\prime \prime}, 82^{\prime \prime}$ and $102^{\prime \prime}$ in the final images, respectively. For the filtering process, regions of the map where the pixel brightness exceeded a threshold defined as twice the standard deviation of the non-zero flux elements in the map were masked from inclusion in the high-pass filter

\footnotetext{
20 See also http://www.mpia-hd.mpg.de/DUNES/.

21 See http://herschel.esac.esa.int/Docs/SPIRE/html/spire_om.pdf for details.
} 
Table 1

Stellar Parameters

\begin{tabular}{|c|c|c|c|c|c|c|c|c|c|c|c|c|}
\hline HIP & HD & Name & $\mathrm{Sp}$ & $\begin{array}{c}d \\
(\mathrm{pc})\end{array}$ & $b$ & $\beta$ & $L / L_{\odot}$ & $\begin{array}{l}T_{\text {eff }} \\
(\mathrm{K})\end{array}$ & $\log g$ & {$[\mathrm{Fe} / \mathrm{H}]$} & $\begin{array}{l}\text { Age } \\
\text { (Gyr) }\end{array}$ & Ref. \\
\hline $171 \mathrm{~A}$ & 224930 & 85 Peg A & G5V & 12.17 & -34.5 & +24.5 & 0.613 & 5600 & 4.51 & -0.66 & $3.1,4.0$ & 1 \\
\hline B & & 85 Peg B & K7V & 12.17 & & & 0.074 & 4200 & 4.77 & -0.64 & & 1 \\
\hline 29271 & 43834 & $\alpha$ Men & G5V & 10.20 & -28.8 & -81.8 & 0.847 & 5591 & 4.46 & 0.08 & $3.4,5.5$ & 2 \\
\hline 49908 & 88230 & & K8V & 4.87 & +52.1 & +35.4 & 0.125 & 4081 & 4.71 & -0.16 & $3.2, \cdots$ & 3,4 \\
\hline 73100 & 132254 & & F7V & 25.11 & +57.2 & +61.4 & 2.831 & 6220 & 4.15 & -0.03 & $1.2,7.2$ & 2 \\
\hline 92043 & 173667 & $110 \mathrm{Her}$ & F6V & 19.21 & +10.4 & +43.4 & 6.141 & 6431 & 4.08 & 0.04 & $0.3,4.7$ & 2 \\
\hline 109378 & 210277 & & G0V & 21.56 & -46.9 & +3.6 & 1.002 & 5540 & 4.39 & 0.22 & $\cdots, \cdots$ & 2 \\
\hline
\end{tabular}

Notes. $b$ and $\beta$ are the galactic and the ecliptic latitude in degrees, respectively. The age column lists two values: from X-ray luminosity and based on the $R^{\prime}(\mathrm{HK})$ index.

References. (1) Bach et al. 2009; (2) Eiroa et al. 2013; (3) Boyajian et al. 2012; (4) Anderson \& Francis 2011.

calculation. Deglitching was carried out using the second level spatial deglitching task, following issues with the clipping of the cores of bright sources using the MMT deglitching method. The two individual PACS scans were mosaicked to reduce sky noise and suppress $1 / f$ stripping effects from the scanning. Final image scales were $1^{\prime \prime}$ pixel $^{-1}$ at $70 \mu \mathrm{m}$ and $100 \mu \mathrm{m}$ and $2^{\prime \prime}$ pixel $^{-1}$ at $160 \mu \mathrm{m}$ compared to native instrument pixel sizes of 3".2 and 6".4. For the SPIRE observation, the small map was created using the standard pipeline routine in HIPE, using the naive mapper option. Image scales of $6^{\prime \prime}, 10^{\prime \prime}$, and $14^{\prime \prime}$ pixel $^{-1}$ were used at $250 \mu \mathrm{m}, 350 \mu \mathrm{m}$, and $500 \mu \mathrm{m}$.

PACS photometry was carried out using two different methods. The first method consisted of estimating PACS fluxes using circular aperture photometry with radii $4^{\prime \prime}, 5^{\prime \prime}$, and $8^{\prime \prime}$ at $70 \mu \mathrm{m}$, $100 \mu \mathrm{m}$, and $160 \mu \mathrm{m}$, respectively. These apertures were chosen in the case of point sources and because they provide the highest signal-to-noise ratio $(\mathrm{S} / \mathrm{N})$ as estimated by the Herschel team $^{22}$ and confirmed by our own analysis (Eiroa et al. 2013). The corresponding beam aperture correction as given in the Herschel technical note PICC-ME-TN-037 was taken into account. The reference background region was usually taken as a ring of width $10^{\prime \prime}$ at a separation of $10^{\prime \prime}$ from the circular aperture size. Nonetheless, we took special care to choose the reference sky region for those objects where the "default" sky was or could be contaminated by background objects, e.g., in the cases of HIP 171 and HIP 109378. Sky noise for each PACS band was calculated from the rms pixel variance of 10 sky apertures of the same size as the source aperture and randomly distributed across the uniformly covered part of the image. Final error estimates take into account a correlated noise factor of 3.7, as estimated by us for the DUNES observations, which is a bit larger than the one given in the technical note PICC-ME-TN-037 (see Eiroa et al. 2013). The second method used to estimate the photometry consisted of using rectangular boxes with areas equivalent to the default circular apertures. However, for extended sources (HIP 29271, HIP 49908, and HIP 92043 at $160 \mu \mathrm{m}$ ), we chose boxes large enough to cover the region where the emission is significant as compared to the background noise. The sky level and sky rms noise for this method were estimated from measurements in 10 fields, selected as cleanly as possible by eye, of the same size as the photometric source boxes. Photometric values and errors take into account beam correction factors. The estimated fluxes from both methods, circular and rectangular aperture photometry, agree within the errors. The SPIRE $250 \mu \mathrm{m}$ flux of HIP 92043 was estimated using the SUSSEXtractor tool.

$\overline{22}$ Technical Note PICC-ME-TN-037 in http://herschel.esac.esa.int.
The synthetic stellar spectra of the six stars were calculated with the PHOENIX/Gaia models (Brott \& Hauschildt 2005), using the stellar parameters given in Table 1 . These spectra were fitted vertically to the optical and near-IR photometry obtained from all available sources. These included the Hipparcos catalog $(B, V, I)$, the Strömgren $u, v, b, y$ photometry (Hauck \& Mermilliod 1998), UKIRT-B ( $\left.V, J, K, L^{\prime}\right), 2 \operatorname{MASS}\left(J, H, K_{s}\right.$; see Cutri et al. 2003; Skrutskie et al. 2006) with quality flags A and B, the JP11 catalog $(J, H, K, L)$, and the WISE W1, W3, and W4 bands (W2 was excluded because of significant flux calibration problems). One of the stars, HIP 171 (=85 Peg), needed special treatment. It is a spectroscopic binary (distance $12.17 \mathrm{pc}$, separation between both components 0.83 or $10.1 \mathrm{AU}$, period $26.3 \mathrm{yr}$, and eccentricity 0.38 ), and a photospheric fit considering both components $\mathrm{A}$ and $\mathrm{B}$ was performed. ${ }^{23}$ For that star, WISE W4 was not used because there are indications that another warm excess that starts around W4 may be present (Koerner et al. 2010).

The method of normalization of the photospheric models to the photometry is explained in detail in Appendix $\mathrm{C}$ of Eiroa et al. (2013). Five subsets of the full SED were chosen to carry out five normalizations, namely, VI+nIR, BVI+nIR, VI+nIR+WISE, nIR+WISE, and VI+nIR+WISE (see Eiroa et al. 2013). A reduced $\chi^{2}$ was computed for each of them. The normalization with the least reduced $\chi^{2}$ was then selected and used to predict the photospheric fluxes $S_{\lambda}$ at the PACS and SPIRE wavelengths.

The predicted photospheric fluxes together with the Herschel measurements are listed in Table 2. The indicated photospheric uncertainties should be taken as lower limits because these do not include the individual uncertainties in each observed point. However, to realistically estimate the actual accuracy of the photospheric predictions, we made additional checks. For two stars, HIP 171 and HIP 49908, we carried out a scaling of the photospheric models with a completely different method, independent of the optical and near-IR photometry. We took advantage of the fact that for these objects the stellar radii, $R_{\star}$, had been measured with high accuracy. Since the distance to the stars, $d$, is also known, this enabled us to compute the "dilution factors" $\left(R_{\star} / d\right)^{2}$ for each star. The PHOENIX/Gaia model photospheres provide the flux density at the stellar surface. Multiplying it by the dilution factor, one directly obtains the flux density measured at Earth. The predictions of the photospheric

\footnotetext{
${ }^{23}$ In fact, HIP $171 \mathrm{~B}$ is likely to have an additional companion $\mathrm{Bb}$ with a mass $\sim 0.1-0.2 M_{\odot}$ (see Bach et al. 2009 and references therein). Furthermore, Schmitt (1997) has suggested the presence of an additional component $C$ more than $1^{\prime}$ away with spectral type M6 and $V=17$ (see also the Washington Double Star catalog).
} 
Table 2

Fluxes and Disk Parameter Estimates

\begin{tabular}{|c|c|c|c|c|c|c|}
\hline HIP & $171^{\mathrm{a}, \mathrm{b}}$ & $29271^{c}$ & $49908^{\mathrm{c}, \mathrm{d}}$ & $73100^{\mathrm{a}, \mathrm{e}}$ & $92043^{\mathrm{c}, \mathrm{f}}$ & $109378^{a}$ \\
\hline$\overline{S_{70}}$ & $\ldots$ & $\ldots$ & $\ldots$ & $14.4 \pm 0.2$ & $48.8 \pm 0.8$ & $\ldots$ \\
\hline$F_{70}$ & $\ldots$ & $\ldots$ & $\ldots$ & $24.7 \pm 3.2$ & $59.0 \pm 3.5$ & $\ldots$ \\
\hline$\chi_{70}$ & $\cdots$ & $\cdots$ & $\cdots$ & $10.3 \pm 3.2$ & $10.2 \pm 3.6$ & $\cdots$ \\
\hline$\chi_{70} / \sigma_{70}$ & $\cdots$ & $\cdots$ & $\cdots$ & 3.2 & 2.8 & $\cdots$ \\
\hline$S_{100}$ & $11.4 \pm 0.2$ & $17.4 \pm 0.2$ & $24.6 \pm 1.0$ & $7.1 \pm 0.1$ & $23.9 \pm 0.4$ & $4.7 \pm 0.1$ \\
\hline$F_{100}(\mathrm{PACS})$ & $11.7 \pm 1.3$ & $17.8 \pm 1.3$ & $22.5 \pm 0.9$ & $13.8 \pm 0.8$ & $30.2 \pm 2.4$ & $8.5 \pm 1.0$ \\
\hline$\chi_{100}$ & $\cdots$ & $\cdots$ & $\ldots$ & $6.7 \pm 0.8$ & $6.3 \pm 2.4$ & $3.8 \pm 1.0$ \\
\hline$\chi_{100} / \sigma_{100}$ & $\cdots$ & $\cdots$ & $\cdots$ & 8.4 & 2.6 & 3.8 \\
\hline$\overline{S_{160}}$ & $4.4 \pm 0.09$ & $6.8 \pm 0.08$ & $9.6 \pm 0.40$ & $2.8 \pm 0.04$ & $9.3 \pm 0.16$ & $1.8 \pm 0.03$ \\
\hline$F_{160}(\mathrm{PACS})$ & $12.5 \pm 2.4$ & $14.4 \pm 2.0$ & $16.0 \pm 1.7$ & $12.5 \pm 1.7$ & $21.9 \pm 3.8$ & $12.4 \pm 1.6$ \\
\hline$\chi_{160}$ & $8.1 \pm 2.4$ & $7.6 \pm 2.0$ & $6.4 \pm 1.7$ & $9.7 \pm 1.7$ & $12.6 \pm 3.8$ & $10.6 \pm 1.6$ \\
\hline$\chi_{160} / \sigma_{160}$ & 3.4 & 3.8 & 3.8 & 5.7 & 3.3 & 6.6 \\
\hline$S_{250}$ & $\ldots$ & $\ldots$ & $\ldots$ & $\ldots$ & $3.8 \pm 0.07$ & $\ldots$ \\
\hline$F_{250}$ (SPIRE) & $\ldots$ & $\ldots$ & $\ldots$ & $\ldots$ & $11.1 \pm 7.2$ & $\ldots$ \\
\hline$\chi_{250}$ & $\ldots$ & $\ldots$ & $\ldots$ & $\ldots$ & $7.3 \pm 7.2$ & $\ldots$ \\
\hline$\chi_{250} / \sigma_{250}$ & $\ldots$ & $\ldots$ & $\ldots$ & $\ldots$ & 1.0 & $\ldots$ \\
\hline$\overline{R_{\text {disk }}}$ & $<6^{\prime \prime}(<760 \mathrm{AU})$ & $8^{\prime \prime}(80 \mathrm{AU})$ & $10^{\prime \prime}(50 \mathrm{AU})$ & $<6^{\prime \prime}(<150 \mathrm{AU})$ & $7^{\prime \prime}(130 \mathrm{AU})$ & $<6^{\prime \prime}(<130 \mathrm{AU})$ \\
\hline$f_{d}$ & $3 \times 10^{-5}$ & $1 \times 10^{-6}$ & $2 \times 10^{-6}$ & $3 \times 10^{-6}$ & $5 \times 10^{-7}$ & $5 \times 10^{-6}$ \\
\hline$\overline{\text { Ref }}$ & 1 & 1,2 & 1,2 & 1 & 1 & 1,2 \\
\hline
\end{tabular}

Notes. Predicted photospheric (“S”), observed ("F”), and excess (“ $\chi$ ”) fluxes and their $1 \sigma$ uncertainties (in mJy), significance of the excesses $(\chi / \sigma)$, disk radius estimates or upper limits inferred from the analysis of the images $\left(R_{\text {disk }}\right)$, and the dust fractional luminosity $\left(f_{d}\right)$.

${ }^{\text {a }}$ Point-like both at $100 \mu \mathrm{m}$ and $160 \mu \mathrm{m}$.

b The only excess source with mispointing around $2 \sigma\left(5^{\prime \prime} \cdot 5\right)$. An additional check of the accuracy of the predicted photospheric fluxes was done by using the radii of components A and B given in Bach et al. (2009), 0.834 and $0.512 R_{\odot}$, and the distance, $d=12.17 \pm 0.33 \mathrm{pc}$, from the Hipparcos parallax $82.17 \pm 2.23$ mas (van Leeuwen 2007). This enabled us to compute the "dilution factors" $\left(R_{\star} / d\right)^{2}$ for each star. The individual PHOENIX model photospheres for A and B were multiplied by the corresponding dilution factors to obtain the flux density measured at Earth, and then added to build the composite final photospheric SED. The uncertainty in each dilution factor was estimated by propagating the errors according to the individual uncertainties in $R_{\star}$ (not given in Bach et al., we assumed it to be 5\%) and $d$ (as indicated above). With this, the predictions for $S_{100}$ and $S_{160}$ are $11.6 \pm 1.2 \mathrm{mJy}$ and $4.5 \pm 0.5 \mathrm{mJy}$, respectively. This is in an excellent agreement with the values given in the table.

${ }^{\mathrm{c}} R_{\text {disk }}$ is an estimate from the deconvolved $160 \mu \mathrm{m}$ image.

${ }^{\mathrm{d}}$ In a similar manner as the one described in note (b), the very accurate stellar radius $\left(0.6415 \pm 0.0048 R_{\odot}\right)$ given by Boyajian et al. (2012) for HIP 49908 and the distance $4.87 \pm 0.01 \mathrm{pc}$ from the Hipparcos parallax 205.21 \pm 0.54 mas (van Leeuwen 2007) were used to compute the dilution factor $\left(R_{\star} / d\right)^{2}$. The PHOENIX model photosphere was scaled by the dilution factor to build the photospheric SED. The uncertainties in the radius and distance were used to estimate the uncertainty in the dilution factor. The results for the predicted fluxes are $S_{100}=24.8 \pm 0.4 \mathrm{mJy}$ and $S_{160}=9.7 \pm 0.15 \mathrm{mJy}$, i.e., within $0.2 \mathrm{mJy}$ from the values listed in the table. ${ }^{\mathrm{e}} F_{70}$ from MIPS (reduction by G. Bryden).

${ }^{\mathrm{f}} F_{70}$ from PACS. The MIPS measurement gives $69.8 \pm 8.9$ mJy (reduction by G. Bryden).

References. (1) Eiroa et al. 2013; (2) Eiroa et al. 2011.

fluxes obtained in this way match very well those from the method described above (see notes $\mathrm{b}$ and $\mathrm{d}$ to Table 2).

Apart from the fluxes, Table 2 gives an estimate of the disk radius $R_{\text {disk. }}$. If the emission at $160 \mu \mathrm{m}$ is extended, then we derive it from the deconvolved image of the source. If it appears point-like, then we give an upper limit on $R_{\text {disk }}$ of $1 / 2$ FWHM at $160 \mu \mathrm{m}$ (as indicated by the "<" sign).

Figure 1 displays the SEDs. It shows all ancillary photometry points, including those not used for the photospheric normalization, such as the $A K A R I 9 \mu \mathrm{m}$ and $18 \mu \mathrm{m}$ fluxes and Spitzer/MIPS $24 \mu \mathrm{m}$ and $70 \mu \mathrm{m}$ data. The best-fit photospheric models are overplotted. The same figure depicts our Herschel PACS and SPIRE measurements and upper limits. In addition, it plots the excess fluxes obtained after the photospheric subtraction.

\subsection{Origin of the Observed Emission}

Table 2 shows that the significance of the $160 \mu \mathrm{m}$ excess detection is larger than $5 \sigma$ for two sources, HIP $73100(5.7 \sigma)$ and HIP $109378(6.6 \sigma)$, while the other four sources only have a significance between $3.3 \sigma$ and $3.8 \sigma$. At this level of confidence, there remains a possibility that some of the cases are false detections due to noise, with a Gaussian probability ranging from $4 \times 10^{-11}$ for HIP 109378 to $1 \times 10^{-3}$ for HIP 92043 .

Note that the probability that these stars do not have any farIR excess at all is much lower, since some of them show excess in the bands other than $160 \mu \mathrm{m}$. Let $P_{i}=1-\operatorname{erf}\left(\left(\chi_{i} / \sigma_{i}\right) / \sqrt{2}\right)$ be the probability that the detection in band $i$ with a significance $\chi_{i}$ is false. For detections in multiple bands $i=1, \ldots, n$ with significances $\chi_{i}$, the probability that all of them are false is

$$
P_{\mathrm{comb}}=1-\operatorname{erf}\left(\frac{\chi_{\mathrm{comb}} / \sigma}{\sqrt{2}}\right)=\prod_{i=1}^{n} P_{i}\left(\chi_{i}\right)
$$

which is an equation to solve for the combined significance in units of the standard deviation, $\chi_{\mathrm{comb}} / \sigma$. For instance, HIP 92043 has a $3.3 \sigma$ excess at $160 \mu \mathrm{m}$, a $2.6 \sigma$ excess at $100 \mu \mathrm{m}$, and a $2.8 \sigma$ excess at $70 \mu \mathrm{m}$. From Equation (1), the 

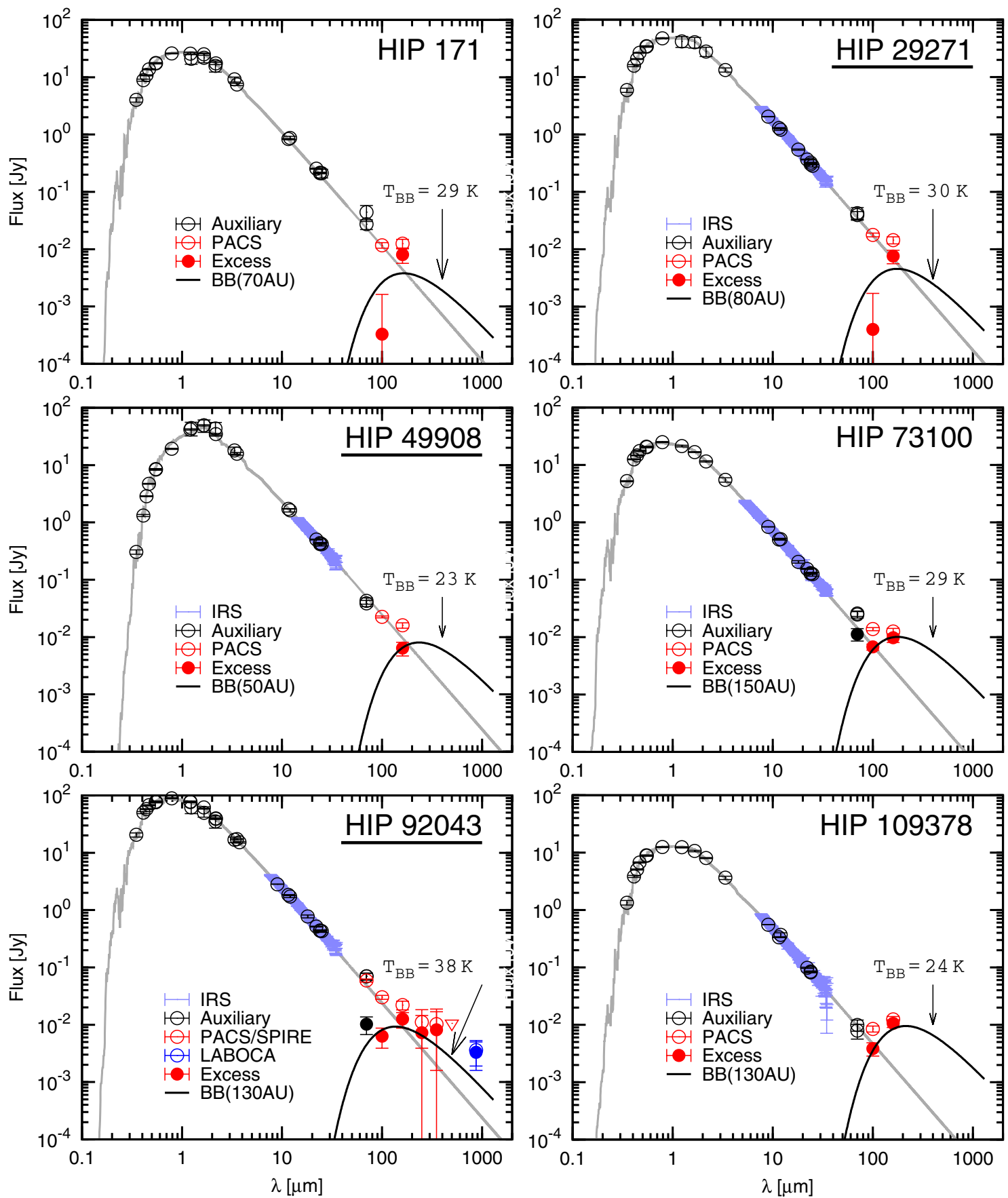

Figure 1. SEDs of six cold disk candidates (names of the resolved ones are underlined). Each plot presents auxiliary photometry (black symbols) and, where available, the Spitzer/IRS spectrum (the light blue line whose thickness reflects error bars). The preferred photosphere is depicted with the gray line. Superimposed are PACS and - where available-SPIRE data (red) and LABOCA (blue) points. The open symbols are total fluxes, the filled ones are excess fluxes (i.e., photosphere subtracted). All symbols are with $1 \sigma$ error bars, which are often smaller than the symbols. The circles are detections, and the triangles are $1 \sigma$ upper limits. Overplotted with a black line is emission from blackbody dust, placed at the distances $R_{\text {disk }}$ given in Table 2.

(A color version of this figure is available in the online journal.)

combined three-band excess significance level is $5.5 \sigma$, and the Gaussian probability that the entire emission of that star is purely photospheric is $4 \times 10^{-8}$.

While it is extremely unlikely that the excess emission is just noise, the SEDs shown in Figure 1, as well as the fact that the DUNES fields are very deep (with a PACS 100/160 on-source time of up to $1440 \mathrm{~s}$ ), raise the question of whether some of the cold disk candidates, or even all of them, may be associated with the galactic background radiation or extragalactic background rather than represent the true circumstellar emission. We deem the former possibility unlikely. Although diffuse cirrus bands are clearly seen around the position of some of the DUNES sources (Eiroa et al. 2013), there is no contamination of this kind in the fields of our six candidates, all of which lie more than $10^{\circ}$ above or below the galactic plane. An unrelated Milky Way object in the line of sight, such as a cold free-floating dwarf, would have to be closer than $1 \mathrm{pc}$ to produce a $30 \mathrm{~K}$ emission at the same flux level as the one observed. A probability of having a trans-Neptunian object in the foreground even in one of the six cases is very small, and for all six just negligible. Note that all 
of the sources, except for HIP 109378, are more than $24^{\circ}$ from the ecliptic (see Table 1).

In contrast, background galaxies pose a serious difficulty. Extragalactic sources unrelated to the targets are clearly visible in most of the fields observed by DUNES, and the SEDs of our six sources peaking longward of $160 \mu \mathrm{m}$ would not be untypical of moderately redshifted galaxies. Even some of the bright, well-resolved disks observed by Herschel were found to be contaminated, as exemplified by 61 Vir (Wyatt et al. 2012). In the DUNES sample of 133 stars, apparent excess emission of seven sources is likely to derive entirely from the extragalactic background (Eiroa et al. 2013, their Appendix D).

Ruling out possible confusion solely by the shape of SEDs is not possible. Taking into account a spread of redshifts and luminosities of potential extragalactic contaminators, one can always attribute a few photometric points to a background galaxy, especially given the uncertainties in the measured fluxes. Alternatively, one could use the morphology of the extended emission to distinguish between the true disks and background sources. Pronounced asymmetry of the emission and a strong offset from the stellar position might favor a background object. Unfortunately, the resolution of Herschel at far-IR wavelengths, the low surface brightness of the cold disk candidates, and complicated background patterns around their position do not allow any definite conclusion on the shape of the observed emission (which appears extended at $160 \mu \mathrm{m}$ for HIP 29271, HIP 49908, and HIP 92043). Even a perfectly symmetric disk centered on the star may easily appear highly asymmetric in observations with low S/Ns. This may be best illustrated by the fact that even a bright, pole-on Vega disk exhibited a bright blob on one side of the star when viewed early with SCUBA (Holland et al. 1998), which was not confirmed later by millimeter observations with higher $\mathrm{S} / \mathrm{N}$ and better resolution (Hughes et al. 2012) or by Spitzer ( $\mathrm{Su}$ et al. 2005) and Herschel (Sibthorpe et al. 2010) observations at shorter wavelengths.

The most direct and reliable way of disentangling the possible confusion toward the cold disk candidates would be to take a second epoch with PACS, trying to find out whether the excess $160 \mu \mathrm{m}$ emission has moved with respect to the $100 \mu \mathrm{m}$ one and the optical position of the star. Unfortunately, all of these observations presented here are single-epoch ones and, even if taking the second epoch were possible, the lifetime of the Herschel mission of less than 4 yr would not be long enough to exclude the background hypothesis, given the proper motion of our candidates (the largest, that of HIP 49908, is $1^{\prime \prime} .4 \mathrm{yr}^{-1}$ ).

We therefore tried yet another method, which was to search the fields around the optical positions of the six stars in catalogs and archives for possible extragalactic objects. For $\mathrm{X}$-ray sources, we accessed the XMM, Chandra, and ROSAT data, using NASA's HEASARC system. ${ }^{24}$ The diameter of the search fields was taken to be $\approx 2^{\prime}$, because of the rather low positional accuracy of the X-ray instruments (e.g., up to 96 " for faint ROSAT sources), so that any object in such a field could be an X-ray counterpart to the Herschel source. Detection of X-ray sources that are inconsistent in their flux with the stars themselves could indicate background galaxies that potentially also contaminate our measurements in the far-IR. Where possible, we have also searched the same fields for optical and near-IR counterparts, using deep images from the Hubble Space Telescope (HST) and VLT/NaCo archives and digitized

\footnotetext{
24 http://heasarc.gsfc.nasa.gov/.
}

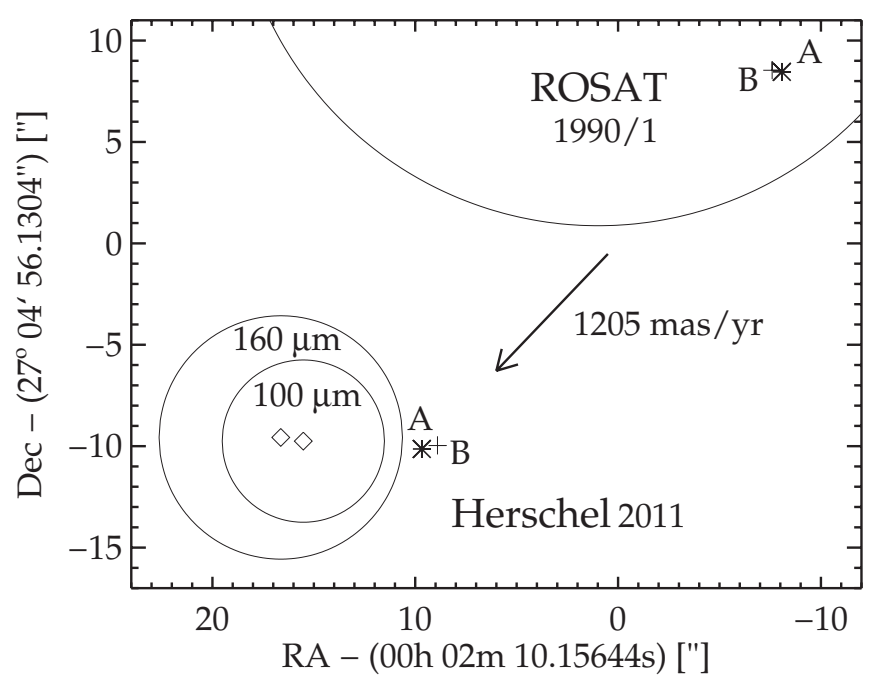

Figure 2. Astrometry of HIP 171. All positions are given in ICRS 2000 relative to the catalog position of the star in 2000. Right upper corner: calculated positions of the components A (asterisk) and B (cross) at the moment of ROSAT observations (between 1990 July 15 and 1991 January 16). Left bottom corner: their positions at the moment of the Herschel observation on 2011 January 17. Since the exact epoch of the ROSAT observation is not known, the proper motion over 6 months can introduce a positional uncertainty of 0.6 . The uncertainty in the position due to that of the proper motion itself (52 mas in $20 \mathrm{yr}$ ) and the parallactic wobble ( $82 \mathrm{mas}$ ) are smaller than the size of the asterisk. ROSAT has detected an X-ray source at $00^{\mathrm{h}} 02^{\mathrm{m}} 11^{\mathrm{s}} .40$ and $+27^{\circ} 05^{\prime} 15^{\prime \prime} .0\left(+1^{\prime \prime} .0,+18^{\prime \prime} .9\right.$ in the relative coordinates used here; lies outside the plot). A circle with a radius of $18^{\prime \prime}$ around that point represents a $1 \sigma$ positional accuracy of ROSAT in this case. The calculated position of the star at the moment of the ROSAT observation coincides with the X-ray source position within $0.77 \sigma$. The diamonds in the left bottom part of the plot show the centers of the $100 \mu \mathrm{m}$ and $160 \mu \mathrm{m}$ emission measured by Herschel in 2011; the circles around them correspond to the FWHMs at those wavelengths. The calculated positions of the star in 2011 and the emission measured by Herschel are consistent with each other to nearly $1 \sigma$.

plate scans from the USNO-A and Digitized Sky Survey (DSS) archives.

HIP 171. The ROSAT catalog (Voges et al. 2000) contains an X-ray source (1RXS J000211.4+270515) with a nominal position $30^{\prime \prime}$ north of the star and a count rate of $0.029 \pm$ 0.011 counts $\mathrm{s}^{-1}$. The All-Sky Optical Catalog of Radio/X-Ray Sources (Flesch \& Hardcastle 2004) reports an optical counterpart $(R=20, B=22)$ to that source with a $69 \%$ likelihood of being a galaxy. However, the digitized plates on which this identification is based are heavily contaminated by the bright nearby star $(V=5.75 \mathrm{mag})$, hampering a detection of such a faint galaxy. Visual inspection suggests that the star HIP 171-being very bright, overexposed, and saturated-was misclassified as an extended source, and hence misidentified as a galaxy. Our interpretation is fully consistent with the study of Schmitt (1997), who finds that the X-ray emission of the X-ray source 1RXS J000211.4+270515 is typical for a late-type star and concludes that either the spectroscopic binary HIP $171 \mathrm{~A}+\mathrm{B}$ or the wide separated later-type companion emits the X-rays. He specifically points out that there is no evidence for an additional background source.

It should also be noted that the ROSAT observation dates back to 1990, whereas the Herschel data were taken in 2011. Given the proper motion of the star $\left(780 \pm 2\right.$ mas $r^{-1}$ in R.A. $\cos ($ decl.) and $-918 \pm 1$ mas $\mathrm{yr}^{-1}$ in decl.), it has moved between the two observations by $24^{\prime \prime}$ toward southeast (P.A. $=140^{\circ}$ ), explaining much of the $30^{\prime \prime}$ offset mentioned above (Figure 2).

HIP 29271 ( $\alpha$ Men). The star, which also has a close M-dwarf true companion at $30 \mathrm{AU}$ (Eggenberger et al. 2007), has been 
associated with an X-ray source (2RXP J061014.3-744510; ROSAT count rate: $0.024 \pm 0.003$ counts $^{-1}$ ).

HIP 49908. HIP 49908 is a flare star that has been detected as an X-ray source too.

HIP 73100. The star is also seen in X-rays (1RXS J145619.7+493753, $0.027 \pm 0.009$ counts $\mathrm{s}^{-1}$ ). The DSS image around HIP 73100 reveals some galaxies that match bright neighboring sources in the PACS field. However, the HST archive image is clean within $8^{\prime \prime}$ from the star.

HIP 92043. HIP 92043 has been detected as a strong X-ray source (2RXP J101122.8+492714, 0.16 counts $\mathrm{s}^{-1}$ ).

HIP 109378. No X-ray source associated with HIP 109378 has been found. In the near-IR, Eggenberger et al. (2007) report no additional sources around HIP 109378 from their VLT/NaCo narrowband data.

In summary, there is an X-ray source in five out of six fields, which is most likely associated with the star. The X-ray count rates are well consistent with the range reported by Schmitt (1997, his Table 2) for his sample of nearby (<13 pc) Sunlike stars. Therefore, the fluxes do not require the presence of background sources. However, it cannot be ruled out. Indeed, most of the galaxies would typically have X-ray fluxes at lower levels. Comparable count rates (Anderson et al. 2007) are primarily expected from active galactic nuclei (AGN). The densities of AGNs at the X-ray flux level in question are on the order of one per square degree (Anderson et al. 2007), rendering multiple alignments in the DUNES fields rather unlikely.

As for the optical/near-IR data, as demonstrated with HIP 73100, contaminating background galaxies can, in principle, be identified via their optical counterparts. We have not found such sources close to the stellar positions. It is still possible though that the catalogs miss optically faint cold galaxies or other objects very close to the Herschel targets.

For all of the stars except for HIP 29271, raw Very Large Array (VLA) radio data are also available from the NRAO Science Data Archive. ${ }^{25}$ However, photometric results have only been published for HIP 49908, with an unconstraining upper limit of $0.08 \mathrm{mJy}$ at $3.6 \mathrm{~cm}$ (Güdel 1992). No extragalactic sources (e.g., AGNs) have been found here.

More VLA data can be found in the NRAO VLA Sky Survey (NVSS; Condon et al. 1998) catalog. It lists a source in positional agreement with HIP 92043 and a tentative continuum flux of $2.7 \mathrm{mJy}$ at $1.4 \mathrm{GHz}(21 \mathrm{~cm})$. The average rms of $0.46 \mathrm{mJy}$ (Condon et al. 1998) for the NVSS suggests an $\mathrm{S} / \mathrm{N}$ of $5-6$. However, inspection of the noise in the VLA field around HIP 92043 suggests the radio detection to be marginal at best (K. Schreyer \& M. Hoeft 2013, private communication). Provided the radio source is real and taking into account that a debris disk does not emit significantly at centimeter wavelengths, both the far-IR excess and the radio counterpart could be caused by the dust emission and the synchrotron emission of a background AGN, respectively. We invoke the expected relation between the far-IR and radio fluxes of a typical AGN (Equation (14) of Condon et al. 1991) to estimate that an AGN mimicking a disk with a far-IR fractional luminosity $f_{d} \approx 5 \times 10^{-7}$ would be consistent with a radio flux $F_{1.49 \mathrm{GHz}} \approx 0.05 \mathrm{mJy}$. Thus, the reported radio flux is off by a factor of 60 , inconsistent with the typical scatter width of only a factor of three observed for the far-IR-to-radio relation for AGNs (Condon et al. 1991). Furthermore, the likelihood of an aleatory alignment of HIP 92043 with an AGN of a

\footnotetext{
25 https://archive.nrao.edu.
}

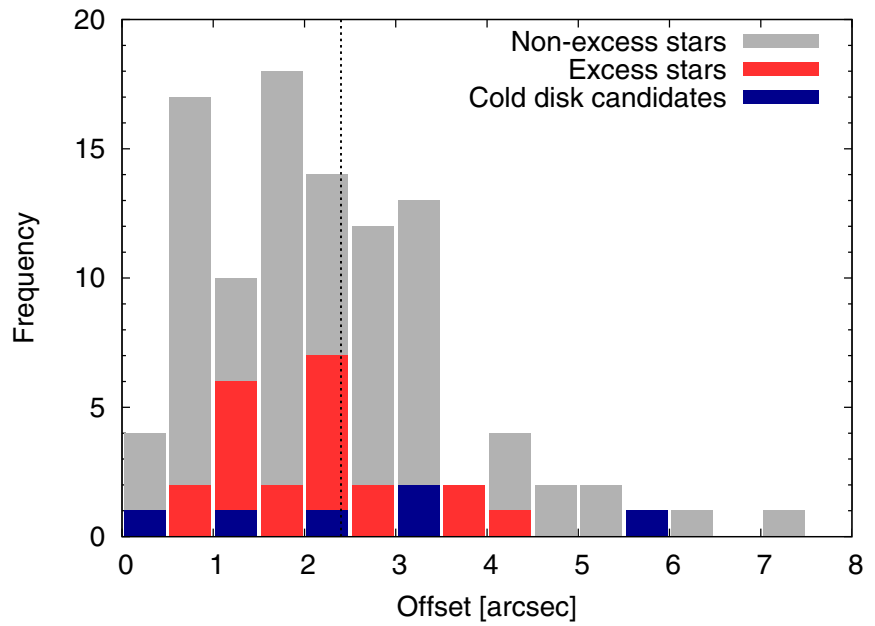

Figure 3. Offsets of the peak of the $100 \mu \mathrm{m}$ emission relative to the optical position of a star for all DUNES sources (gray bars), excess stars excluding cold disk candidates (red), and cold disk candidates (blue). One object (HIP 28442) with an anomalous offset of 10'.5 was left out from the plot. The mean APE of Herschel/PACS is shown with a dashed vertical line.

(A color version of this figure is available in the online journal.)

plausible radio brightness is low. From the statistics presented in Condon et al. (1998), there should be about 100 sources per square degree with $F_{1.4 \mathrm{GHz}}$ in the range from $1 \mathrm{mJy}$ to $10 \mathrm{mJy}$. Within the synthesized FWHM of $45^{\prime \prime}$ for the NVSS, we would therefore expect 0.01 such sources on average. HIP 92043 could hence be one of at most two expected sources in the DUNES sample aligned with AGNs of that magnitude. In summary, the marginality of the detection, the inconsistency between the farIR and radio fluxes, as well as the low density of AGNs at the brightness level in question all render the hypothesis of a coincidental alignment of HIP 92043 with an AGN less likely than that of a classical debris disk plus a spurious radio signal.

Apart from the direct search for possible background galaxies, we can invoke statistical arguments.

1. For the cold disk candidates, the mean offset between the optical position of a star and the peak of the $100 \mu \mathrm{m}$ emission is as small as $2^{\prime \prime} 6$. This is consistent with the Herschel $1 \sigma$ absolute pointing error (APE) of $2.44{ }^{26}$ The distribution of the offsets among the cold disk candidates is also consistent with the distribution of offsets for other DUNES stars (Figure 3). Five out of six have offsets $\leqslant 3$ ".2, and only HIP 171 has 5".5. However, even the latter is just a $\sim 2 \sigma$ outlier, and thus nothing extraordinary, especially for a binary. It is known that the binarity may cause photometric shifts and decrease the accuracy of the proper motion determination. Note that there are five other non-excess sources in the DUNES sample with offsets $>5^{\prime \prime}$ (Eiroa et al. 2013, their Section 5.3).

2. For three sources without an excess at $100 \mu \mathrm{m}$ (HIP 171, HIP 29271, HIP 49908), the measured flux at $100 \mu \mathrm{m}$ is consistent with the photospheric prediction (mean deviation of $1.4 \mathrm{mJy}$, well within the flux uncertainties), strengthening the conclusion that the $100 \mu \mathrm{m}$ emission indeed comes from the star.

3. The mean offset between the peaks of the $160 \mu \mathrm{m}$ emission and the $100 \mu \mathrm{m}$ one is 1 .". 8 , so that the probability that the $160 \mu \mathrm{m}$ emission and the $100 \mu \mathrm{m}$ one are associated with each other is high.

26 This is the APE for the second period of Herschel observations with scan maps, see http://herschel.esac.esa.int/twiki/bin/view/Public/SummaryPointing. 
These arguments are statistical and cannot be considered as evidence for the circumstellar nature of the emission. Yet we can make probabilistic estimates. We assume that to be misinterpreted as a cold disk, a center of a background galaxy should be located within a "spot" with a diameter equal to the FWHM of the PACS instrument at $160 \mu \mathrm{m}$, i.e., 12". The area of a circle of that diameter is $0.0314 \mathrm{arcmin}^{2}$. Next, we estimate the expected density of galaxies at a level of $6 \mathrm{mJy}<F(160 \mu \mathrm{m})<$ $13 \mathrm{mJy}$, which corresponds to the excess flux range of the cold disk candidates at that wavelength (see Table 2). We draw on the study by Berta et al. (2011) that derived the galaxy counts from several Herschel surveys, including GOODS and PEP. Their results are also supported by the most recent analysis of the Herschel/PACS data from the DEBRIS survey (Sibthorpe et al. 2013), with the caveat that the comparison has only been done at $100 \mu \mathrm{m}$ and for relatively bright sources. Based on Figure 7 of Berta et al. (2011), we have estimated the density number of sources in the 6-13 mJy range to be about 5500 sources per square degree (1.53 sources per square arcmin). This density is almost the same as the cumulative density of all galaxies brighter than $6 \mathrm{mJy}$ (the $d N / d S$ slope steepens above $10 \mathrm{mJy}$, see Figure 7 in Berta et al. 2011). To estimate the likelihood of a coincidental alignment in the DUNES fields, we assume that the source density is representative of any point of the sky, so in an FWHM spot we will have $1.53 \times 0.0314 \approx 0.048$ sources, implying the probability of a coincidental alignment of a given cold disk candidate with a galaxy of $p=4.8 \%$. We now consider the whole DUNES sample of $N=133$ stars. The mean number of false detections is simply $N p=6.4$, meaning that six or seven sources in the sample are likely to be background objects. However, as mentioned above, as many as seven of such sources seem to have already been identified (see Section 5.3 of Eiroa et al. 2013). The binomial probability that six more sources (namely, our cold disk candidates) are all galaxies as well is just $1.2 \%$. Nevertheless, the chance that at least one of our six is an unrelated object is $31 \%$. Conversely, there is a $69 \%$ chance that all of them are true disks.

The above estimates are conservative for two reasons. First, we used the density of galaxies brighter than $6 \mathrm{mJy}$ at $160 \mu \mathrm{m}$. This density, and the resulting $p=4.8 \%$, was then applied to all six candidates, although most of them are brighter than $6 \mathrm{mJy}$. Taking into account the excess flux of individual disks (from $6.4 \mathrm{mJy}$ for HIP 49908 to $12.6 \mathrm{mJy}$ for HIP 92043) would reduce the probabilities. For example, the probability $p$ for 8 mJy sources such as HIP 171 and HIP 29271 is only $2.3 \%$. Second, DUNES observations were done at a different depth, with the on-source time ranging between $180 \mathrm{~s}$ and $1440 \mathrm{~s}$. However, a $180 \mathrm{~s}$ observation would not be enough for a $3 \sigma$ detection of a $6 \mathrm{mJy}$ source. Specifically, a $3 \sigma$ detection would only be possible for $\geqslant 11.1 \mathrm{mJy}$ sources at $180 \mathrm{~s}, 7.8 \mathrm{mJy}$ at $360 \mathrm{~s}, \ldots, 4.5 \mathrm{mJy}$ at $1440 \mathrm{~s}$. This means that the expected number of false detections in the whole DUNES sample, which we computed as $N p(>6 \mathrm{mJy})$, where $N=133$ and $p(>6 \mathrm{mJy})=4.8 \%$ is again an overestimate. If, for instance, we did not count the $180 \mathrm{~s}$ fields, or applied $p(>11.1 \mathrm{mJy})$ to them, then the resulting number would be smaller.

More accurate probability estimates, involving the brightness of individual sources and different integration times, would not make much sense because of a number of additional uncertainties. The actual diameter of the "alignment spot" that goes into the estimate of $p$ may be smaller than the FWHM as assumed here. Besides, the density of galaxies in the DUNES fields may differ somewhat from those in GOODS and other cosmological surveys. Even more importantly, the density of galaxies at the brightness level in question varies from one individual DUNES field to another-for instance, the field around HIP 171 is cleaner than that around HIP 92043. We note, however, that the density adopted above agrees reasonably well with the number of background sources at the brightness level of the cold disk candidates and with a similar $100 / 160 \mu \mathrm{m}$ color, which are seen in the $2^{\prime} \times 2^{\prime}$ fields around the positions of the six candidates. A detailed study of the contamination within the DUNES fields is in progress (C. del Burgo et al., in preparation).

We conclude that, most likely, our set of six candidates contains both real disks and unrelated background galaxies. We also emphasize that HIP 73100 and HIP 92043 almost certainly host true debris disks, evident in the $70 \mu \mathrm{m}$ excess, regardless of whether their cold components are real. Beyond that, we do not see any possibility to distinguish between the real cases and "false alarms," and observational prospects to find final answers are discussed in Section 5. In what follows, we assume that some of the cold disk candidates do represent true circumstellar disks. In light of the observational uncertainties described above, detailed modeling of the individual objects in our set would not be warranted. Therefore, the subsequent analysis seeks a possible qualitative explanation for the phenomenon. Conceivable scenarios are then checked against numerical simulations.

\section{GRAIN SIZES AND COMPOSITION}

\subsection{Blackbody Grains}

We start by getting a handle on the dust temperature in the cold disks. To this end, we have computed blackbody emission by placing the emitting material at a distance $R_{\text {disk }}$ from each star. The results were then scaled vertically to match the excess fluxes at $100 \mu \mathrm{m}$ and $160 \mu \mathrm{m}$. The results are shown in Figure 1; the blackbody temperature as indicated in the panels ranges from $23 \mathrm{~K}$ (HIP 49908) to $38 \mathrm{~K}$ (HIP 92043).

HIP 109378 is nicely consistent with blackbody grains. For HIP 73100 and HIP 92043, there is an excellent agreement at $100 \mu \mathrm{m}$ and $160 \mu \mathrm{m}$. However, those stars reveal an $\sim 3 \sigma$ excess at $70 \mu \mathrm{m}$ (see notes to Table 2), which may be indicative of an additional inner dust component. HIP 49908 provides weak constraints, as the excess was only found at $160 \mu \mathrm{m}$. Finally, for HIP 171 and HIP 29271 the rise of the excess flux from 100 to $160 \mu \mathrm{m}$ is steeper than blackbody. However, as the deviation from the blackbody model is $<2 \sigma$, this may not be genuine. If it is, then this would either imply "subthermal" dust (colder than blackbody) or indicate background galaxies. These two objects might particularly be interesting for ALMA follow-ups, as discussed in Section 5.

\subsection{Compact Grains of Pure Materials}

The nearly blackbody temperatures of grains require them either to be large or, if they are small, to have low absorption in the visible. In this section, we investigate which of these two options appears more probable. More generally, we try to find out which kind of material the cold disks should be composed of to reproduce the observed thermal emission.

We have chosen four material compositions: astrosil (Draine 2003b, 2003c), olivine (Fabian et al. 2001), crystalline ice at $-60^{\circ} \mathrm{C}$ (Warren 1984), and amorphous ice (Li \& Greenberg 1998). This choice is motivated by the fact that silicates 
are traditionally assumed as a reference material composition in debris disk modeling, while ice-rich material can also be expected, considering that all cold disks have large radii ( $\sim 100 \mathrm{AU})$. For instance, Lebreton et al. (2012) demonstrated that icy inclusions markedly improve the fits to the SED of a bright, resolved debris disk of HD 181327. Direct evidence for ices comes from the solar system studies. Indeed, the surfaces of many trans-Neptunian objects contain significant amounts of ice (Barucci et al. 2011). Many large ones have geometric albedos well in excess of 50\%. For instance, Haumea's albedo is 70\%-75\% (Lellouch et al. 2010), Makemake includes a bright terrain with an albedo of $\sim 80 \%$ (Lim et al. 2010), Sedna and $2010 \mathrm{EK}_{139}$ have $32 \%$ and 25\%, respectively (Pál et al. 2012). Some scattered EKBOs have albedos of up to $85 \%$ (Santos-Sanz et al. 2012), while cold classical EKBOs, despite their smaller sizes, still have an average albedo of $17 \%$ (Vilenius et al. 2012). It is natural to expect that dust released from the surfaces of such objects would be highly reflective too.

As for sizes, we selected two grain radii: $10 \mu \mathrm{m}$ and $1 \mathrm{~mm}$. The former choice is a proxy for a typical cross sectiondominating size expected from collisional models of debris disks (e.g., Wyatt et al. 1999; Wyatt \& Dent 2002; Krivov et al. 2006; Thébault \& Augereau 2007). The latter choice is meant to show the emission of grains with a size parameter (for the Herschel wavelengths) exceeding unity. At this point, we intentionally limit ourselves to single sizes, in order to get a clearer understanding of the simulation results in Section 4 that involve size distributions.

The absorption efficiency $Q_{\text {abs }}$ for all four materials and two sizes, calculated with a standard Mie routine valid for homogeneous, compact spheres (Bohren \& Huffman 1983), is plotted in Figure 4 with solid lines. From these curves, we can expect icy grains of a given size at a given distance from a star to be colder than silicate ones. This is because icy grains, even millimeter-sized ones, have lower absorption efficiencies in the visible (where the stellar flux peaks). At the same time, the absorption efficiency at far-IR wavelengths (where thermal emission of dust peaks) is comparably high for all materials, providing efficient cooling.

With these materials and sizes, we have calculated dust emission for two selected candidates (Figure 5). One is the "clean" case of HIP 109378, where there is a clear, point-like, excess emission at PACS/100 and PACS/160 $\mu \mathrm{m}$ (Eiroa et al. 2011). Based on the fact that the disk is unresolved, the upper limit on the disk radius is $\sim 130$ AU. Another case is HIP 92043, which offers the best-sampled SED. Here, excesses were found in the PACS $/ 70 \mu \mathrm{m}$ band (and previously in MIPS/70), and in the PACS $/ 100 \mu \mathrm{m}$, PACS $/ 160 \mu \mathrm{m}$, and SPIRE/250 $\mu \mathrm{m}$ bands. The target was not detected in the 350 and $500 \mu \mathrm{m}$ SPIRE bands. Finally, there was a marginal (slightly over $2 \sigma$ ) detection by APEX/LABOCA at $870 \mu \mathrm{m}$ (R. Liseau 2011, private communication). The emission at $70 \mu \mathrm{m}$ and $100 \mu \mathrm{m}$ is point-like, but extended at $160 \mu \mathrm{m}$, and we interpret it as stemming from the disk. Then, the deconvolved brightness profiles suggest a disk radius of $\sim 130 \mathrm{AU}$. Since the excess fluxes at $70 \mu \mathrm{m}$ and $160 \mu \mathrm{m}$ are separated by a lower excess flux at $100 \mu \mathrm{m}$, and the images at $70 \mu \mathrm{m}$ and $160 \mu \mathrm{m}$ show a different appearance of the emission (point-like versus extended), it is likely that the $70 \mu \mathrm{m}$ excess derives from an additional component, i.e., an unresolved inner debris disk. If true, the potential cold disk should be associated mostly with the $160 \mu \mathrm{m}$ emission.

Figure 5 overplots the expected thermal emission from fiducial disks of equal-sized grains with different optical properties,
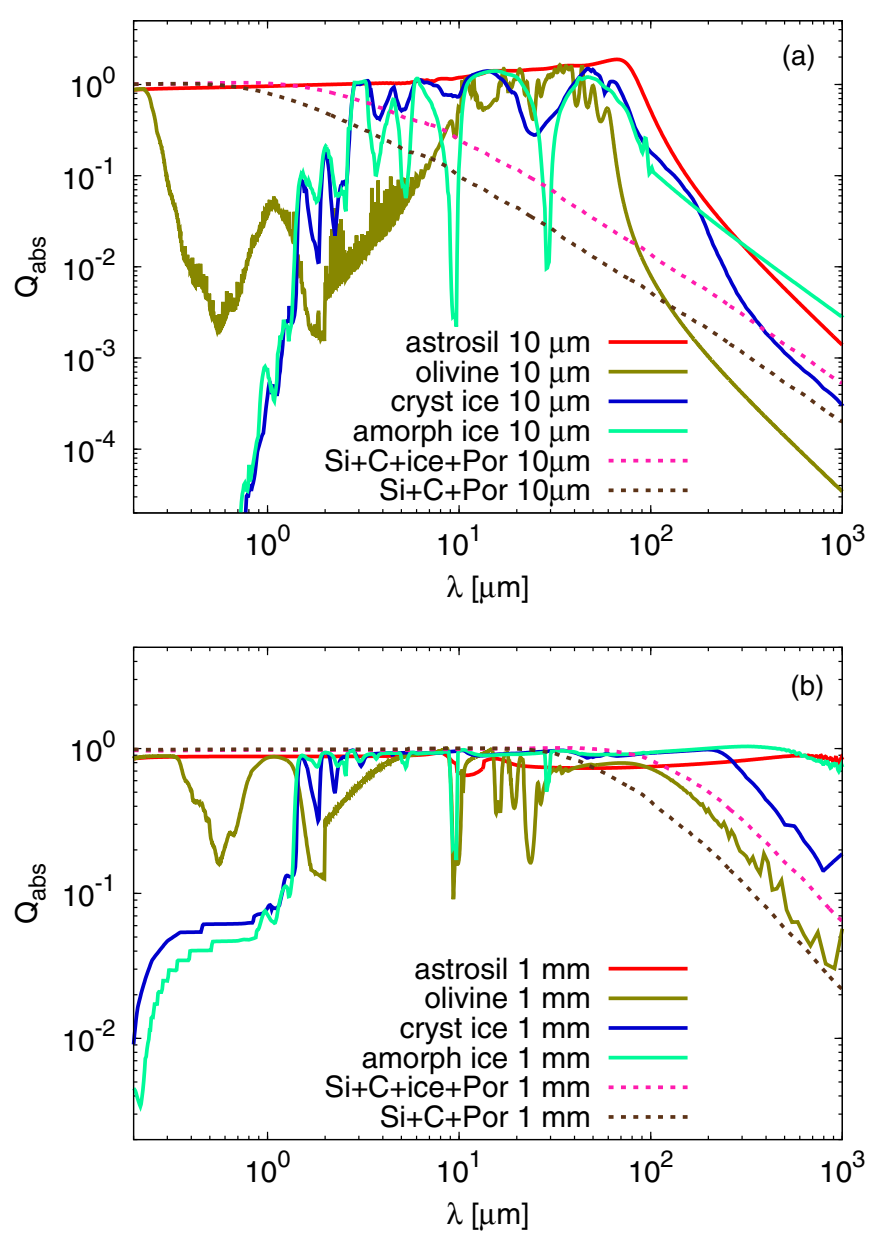

Figure 4. Absorption efficiency for compact grains of single materials (solid lines) and for two advanced grain models (dashed lines). Grain radii: (a) $10 \mu \mathrm{m}$ and (b) $1 \mathrm{~mm}$.

(A color version of this figure is available in the online journal.)

placed at a distance of $130 \mathrm{AU}$ from a G0V (HIP 109378) and F6V (for HIP 92043) star and assumed to be in thermal equilibrium. For HIP 109378, a comparison of the modeled curves and the data points clearly demonstrates that grains smaller than $\sim 10 \mu \mathrm{m}$ - those that are predicted by collisional models_-yield emission that is much too warm to be consistent with the observed fluxes, irrespective of the dust composition. A rough agreement with the observations can only be achieved for larger grains $(\gtrsim 1 \mathrm{~mm})$. Constraints on the material composition are difficult to place. Similar conclusions can be drawn for two other sources, HIP 171 and HIP 29271, not shown in the figure.

For HIP 92043, the conclusions are less certain. However, a conclusion on the prevalence of large grains can also be drawn here if, as argued above, the $70 \mu \mathrm{m}$ excess originates from an unresolved inner component, physically separated from the bona fide cold disk. Again, constraints on the material composition are much weaker than those on sizes. Although using amorphous ice provides a better agreement with the submillimeter point, this should not be overinterpreted, as the LABOCA detection is only marginal. The case of HIP 73100, not shown in the figure, is very similar to HIP 92043.

\subsection{Porous Grains of Material Mixtures}

Real dust grains in various cosmic environments, including circumstellar disks, are expected to be composed of material 
HIP 109378, $10 \mu \mathrm{m}$ grains

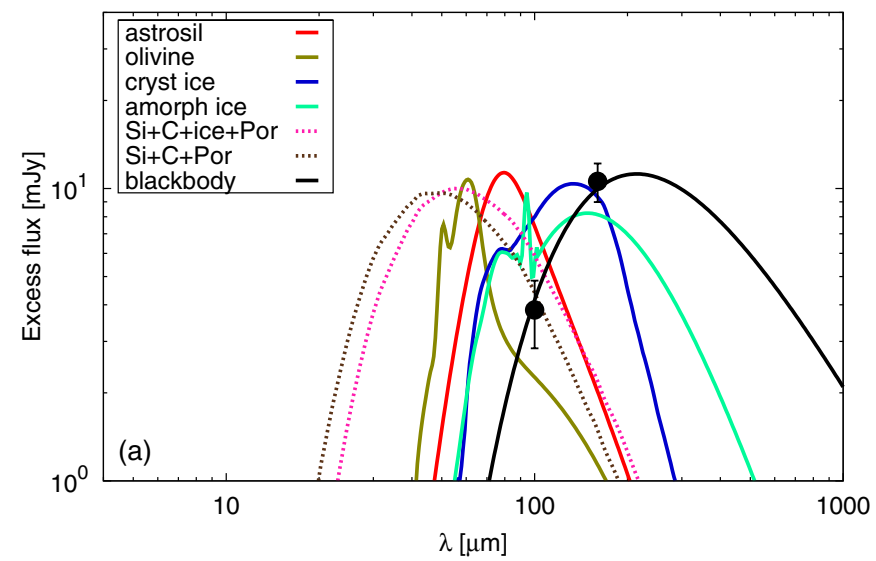

HIP 92043, $10 \mu \mathrm{m}$ grains

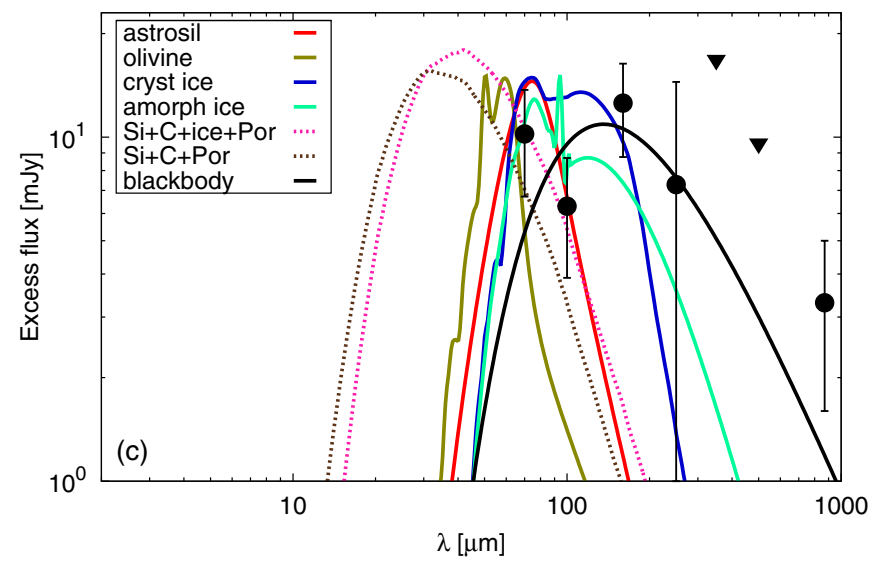

HIP 109378, 1 mm grains

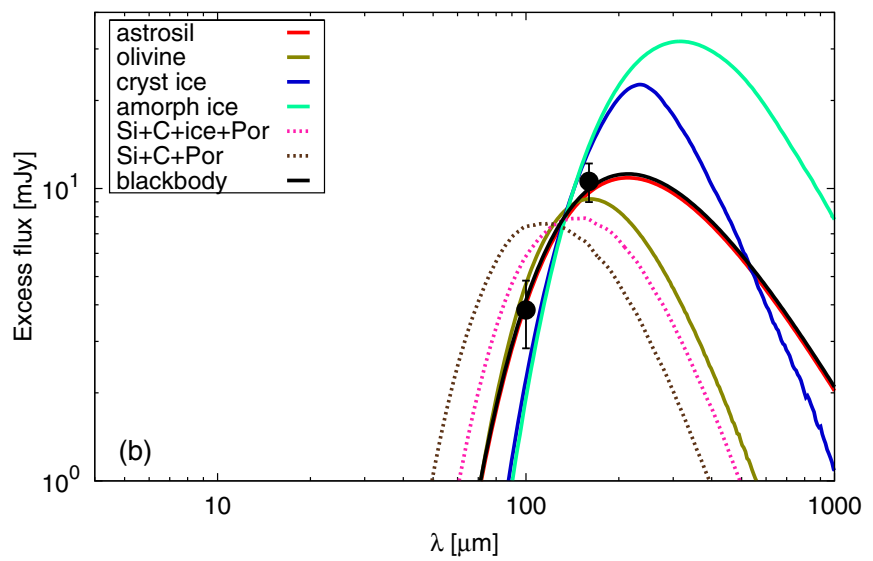

HIP 92043, $1 \mathrm{~mm}$ grains

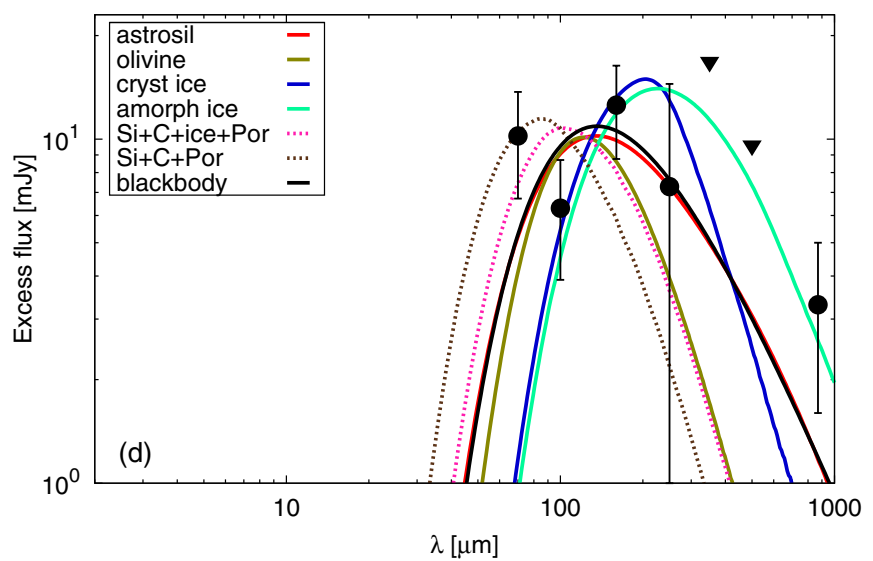

Figure 5. Excess emission of HIP 109378 (top) and HIP 92043 (bottom). The stellar photosphere is subtracted. The filled circles with $1 \sigma$ error bars are PACS/70, PACS /100, PACS/160, SPIRE/250, and LABOCA/870 excess fluxes. The upside-down triangles are the $1 \sigma$ upper limits (SPIRE/350 and SPIRE/500) for HIP 92043. The colored lines are the same as in Figure 4 and show which emission is expected from dust grains of $10 \mu \mathrm{m}$ (left panels) and $1 \mathrm{~mm}$ in radius (right panels), made of several pure materials (solid lines) and mixtures (dashed). For comparison, blackbody emission is shown as a black thick curve. All curves are roughly scaled vertically to the level of the PACS/100 and /160 and, for HIP 92043, also SPIRE/250 points.

(A color version of this figure is available in the online journal.)

mixtures, to have some degree of porosity, and to have a complex morphology, for instance to acquire icy mantles around silicate or organic refractory cores (e.g., Preibisch et al. 1993). Ice mantle growth and dust coagulation are very common physical processes in the interstellar medium (ISM) where the resulting fluffy, porous dust grains show emission enhancements toward the far-IR (del Burgo et al. 2003 and references therein). Multicomponent fitting that includes core-mantle grains has also been commonly done for protoplanetary disks, which represent the debris disk progenitors (e.g., White et al. 2000).

To test how strong the effects of more advanced grain models could be and what impact on the observed emission they may have, we tried two models from Lebreton et al. (2012). The first one provided the best fit to the SED of HD 181327. It consists of $7 \%$ volume fraction of ACAR carbon from Zubko et al. (1996), $3.5 \%$ astrosil from Draine (2003a), 24.5\% amorphous water ice from Li \& Greenberg (1998), and 65\% vacuum to mimic porosity. The bulk density is $0.553 \mathrm{~g} \mathrm{~cm}^{-3}$. Another model provided the "coldest" emission in the case of HD 181327. It has $3.3 \%$ carbon, $1.7 \%$ astrosil, no ice, but a high porosity $(95 \%$ of vacuum) and a low density, $0.123 \mathrm{~g} \mathrm{~cm}^{-3}$. The refractive indices of both mixtures were computed using the Bruggeman mixing rule (Bohren \& Huffman 1983), and the Mie theory was used to calculate the absorption efficiencies.
The absorption efficiencies and SEDs for the same two cold disk candidates are shown with dashed lines in Figures 4 and 5, respectively. Indeed, the effects are seen to be strong, but-in contrast to HD 181327—go in the wrong direction: the SEDs for these two models are warmer, not colder, than those discussed before. This is because the porous grains absorb efficiently where the stellar flux peaks, while these are poor emitters in the IR (Figure 4). As a consequence, the porous grains used here are hotter (at a same distance, which is fixed), and the SED is shifted to the blue.

Nonetheless, this is not to say that realistic grain models are unable to make the dust colder. In fact, the opacities and temperatures of such grains can be both higher and lower than those of compact mono-material ones, depending on the chemical composition, sizes and number of constituent monomers, degree of porosity, wavelength ratio between the maxima of the stellar and dust emission, and other factors (see, e.g., Kimura et al. 1997). Also, the results of the Mie calculations may (Mukai et al. 1992) or may not (Stognienko et al. 1995) be accurate enough. Finally, the mechanical properties (e.g., critical fragmentation energy) of non-ideal grains are expected to be different from those of compact ones (e.g., Love et al. 1993; Stewart \& Leinhardt 2009; Shimaki \& Arakawa 2012). Therefore, for self-consistency, we would have to take porosity 
into account in the collisional modeling performed in Section 4. Taking into account the paucity of the observational data and the as yet unconfirmed circumstellar nature of the "cold emission" of our candidates, we defer including advanced grain models to future studies and do not consider them in the rest of the paper.

\section{POSSIBLE SCENARIOS}

Having concluded that the cold disks should be depleted in small grains, in this section, we seek a possible explanation for that. We try to find a dynamical regime for a debris disk which would simultaneously provide the flux at an observed level and have a cross section peaking at larger sizes.

The simulations described below were performed with our collisional code ACE (Krivov et al. 2005, 2006, 2008; Löhne et al. 2012) and thermal emission utility SEDUCE (Müller et al. 2010). These tools have been successful in reproducing SEDs and resolved images of many other debris disks, ranging from the archetype debris disk around Vega (Müller et al. 2010) to debris disks of the DUNES program such as HD 207129 (Löhne et al. 2012).

The collisional code ACE numerically solves the kinetic equation with various gain, loss, and transport mechanisms to evolve a disk of solids in a broad range of sizes (from smallest dust grains to planetesimals), orbiting a star under the combined action of gravity, direct radiation pressure, and drag forces and experiencing non-elastic collisions. The code implements a three-dimensional model with masses, periastron distances, and eccentricities as phase-space variables. It assumes the disk to be rotationally symmetric and averages over the inclinations of the constituent particles within the semi-opening angle of the disk. Collision outcomes are simulated as follows. The mechanical strength of the disk material is described by the critical energy for fragmentation and dispersal:

$$
Q_{D}^{*}(s)=A_{s}(s / 1 \mathrm{~m})^{b_{s}}+A_{g}(s / 1 \mathrm{~km})^{b_{g}}
$$

where $s$ is the radius of the target. Unless otherwise stated, we choose $A_{s}=1.0 \times 10^{6} \mathrm{erg} \mathrm{g}^{-1}, b_{s}=-0.37, A_{g}=$ $2.0 \times 10^{6} \mathrm{erg} \mathrm{g}^{-1}$, and $b_{g}=1.38$, which is close to the values used by many authors (see, e.g., Davis et al. 1985; Holsapple 1994; Paolicchi et al. 1996; Durda \& Dermott 1997; Durda et al. 1998; Benz \& Asphaug 1999; Kenyon \& Bromley 2004). For each collision, ACE first checks if the impact energy, $E_{\text {imp }}\left(m_{\mathrm{p}}, m_{\mathrm{t}}\right)$, exceeds the critical one, $E_{\text {crit }}\left(m_{\mathrm{p}}, m_{\mathrm{t}}\right) \equiv$ $\left(m_{\mathrm{p}}+m_{\mathrm{t}}\right) \times Q_{D}^{*}\left(m_{\mathrm{p}}+m_{\mathrm{t}}\right)$, where $m_{\mathrm{p}}$ and $m_{\mathrm{t}}$ are the masses of a projectile and target, respectively. If it does, then the collision is treated as disruptive. If not, then further checks are being done to determine whether the collision is cratering (the projectile is disrupted, but the target is only cratered), bouncing (both impactors are cratered), or sticking (the two impactors merge into one).

For the purposes of this study, we made a number of further improvements to ACE. All of these do not affect the modeling results as long as the relative velocities exceed a few tens of $\mathrm{m} \mathrm{s}^{-1}$, but they provide a more accurate treatment at lower velocities. First, the simulations are now being done over a logarithmically spaced (instead of a linearly spaced) eccentricity grid. Second, we have changed the prescriptions for the outcomes of grain-grain collisions in the cratering, bouncing, and sticking regimes. These are implemented in a model that approximately conforms to a semi-empirical model of Güttler et al. (2010).

\subsection{Disks in the Transport-dominated Regime}

One possibility to reduce the proportion of small grains is to assume that the disks in question are transport dominated rather than collision dominated (Krivov et al. 2000; Wyatt 2005), meaning that small grains are displaced inward from their birth location by Poynting-Robertson (P-R) drag before they get lost to collisions. This can indeed be expected, since this regime is achieved at normal optical depths lower than roughly $v_{K} / c$, where $v_{K}$ is the local Keplerian speed (Kuchner \& Stark 2010), which is consistent with low $f_{d}$ of the cold disks. In that case, the dominant size of the grain cross section shifts to larger values (Vitense et al. 2010; Wyatt et al. 2011), which is exactly what is needed. However, we must check whether our disks are tenuous enough to fall into this regime. Besides, even if they are, an additional difficulty may arise, as smaller grains are not eliminated from the system. Instead, they drift inward and heat up very efficiently, since they are small and close to the star. As a result, they could produce warm emission, making the SEDs inconsistent with those of the cold disks.

To check this scenario, we ran ACE with a setup that matches the conditions of the HIP 109378 disk. The material was initially placed into a narrow ring with a half-width of $10 \mathrm{AU}$ centered at $130 \mathrm{AU}$. We assumed the disks to be composed initially of planetesimals up to $s_{\max } \approx 1 \mathrm{~m}$ in radius with a differential size distribution slope of 3.7 (this slope is expected for collisional equilibrium in the strength regime; O'Brien \& Greenberg 2003). The minimum size was set to $s_{\min }=3 \mu \mathrm{m}$, but the assumed $s_{\min }$ does not affect the final results because the system immediately finds the equilibrium distribution of grains at such sizes. We tried different initial total disk masses: $0.1 M_{\oplus}, 10^{-3} M_{\oplus}$, and $10^{-5} M_{\oplus}$. Henceforth, we refer to these three as "highmass," "mid-mass," and "low-mass" disks, respectively. Note that the results of these simulations are almost independent of the maximum size $s_{\max }$ chosen. It only has to be large enough for the largest bodies not to be involved in the collisional cascade by the time when the distribution of material at dust sizes reaches a quasi-steady state (Löhne et al. 2008), and $1 \mathrm{~m}$ suffices for the disks considered here. Choosing larger $s_{\max }$ would only slow down the simulations. The results obtained here can readily be applied to larger $s_{\max }$, except that an up-scaling of the disk mass is required. For instance, if bodies as large as $100 \mathrm{~km}$ are present, then the total mass of the high-mass disk will be about $30 M_{\oplus}$ instead of $0.1 M_{\oplus}$. The planetesimal disk was assumed to be moderately stirred to eccentricities $e \sim 0.1$ and inclinations $I \sim 0.05$ (energy equipartition). We took a homogeneous mixture of astrosilicate (Draine 2003b, 2003c) and amorphous water ice ( $\mathrm{Li} \&$ Greenberg 1998) in equal volume fractions $\left(\rho_{d}=2.35 \mathrm{~g} \mathrm{~cm}^{-3}\right)$. The optical constants of the mixture were calculated using the effective medium theory with the Bruggeman mixing rule. The mechanical strength of the disk material was set as described above.

We evolved all three disks until a quasi-steady state (Löhne et al. 2008) was reached: after $\sim 0.5 \mathrm{Myr}$ for the high-mass disk, $\sim 50 \mathrm{Myr}$ for the mid-mass disk, and $\sim 5 \mathrm{Gyr}$ for the low-mass disk. This reflects the property that the collisional timescales approximately go as a reciprocal of the disk mass (Krivov et al. 2008). The timescales listed above should not be misinterpreted as physical time of collisional evolution of the disks. Instead, these are merely the "relaxation" times for the fiducial collisiondriven systems.

The simulation results are presented in Figures 6 and 7 that plot the size distributions and the radial profiles of the optical depth, respectively. Obviously, the high-mass disk is collision 


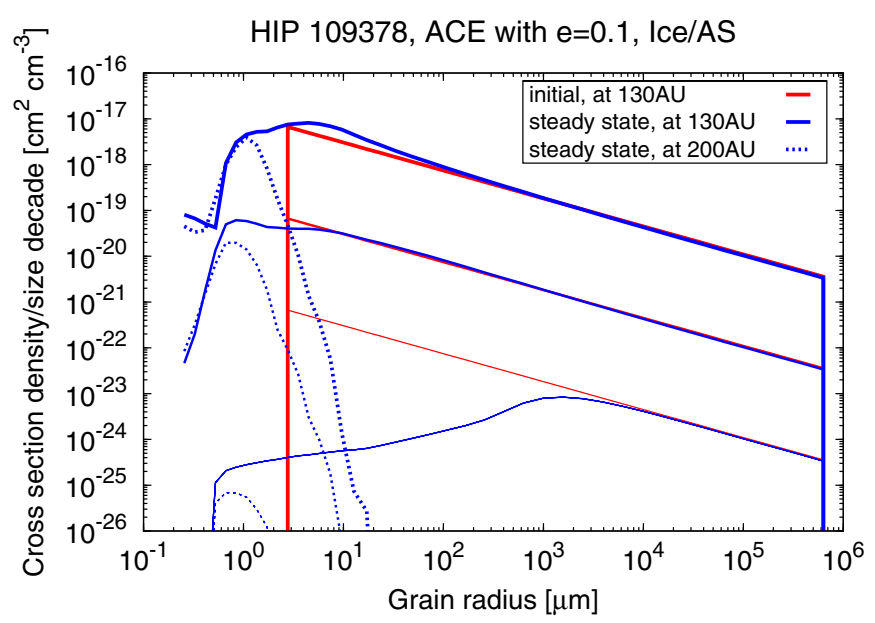

Figure 6. Simulated evolution of the size distribution of "classical" debris disks in an attempt to match the conditions of HIP 109378. Thick, medium, and thin lines: disks with initial total masses of $0.1 M_{\oplus}, 10^{-3} M_{\oplus}$, and $10^{-5} M_{\oplus}$ in the bodies smaller than $1 \mathrm{~m}$, respectively. Red solid lines: assumed initial size distributions; blue solid lines: final distributions at $130 \mathrm{AU}$; blue dashed lines: final distributions at $200 \mathrm{AU}$.

(A color version of this figure is available in the online journal.)

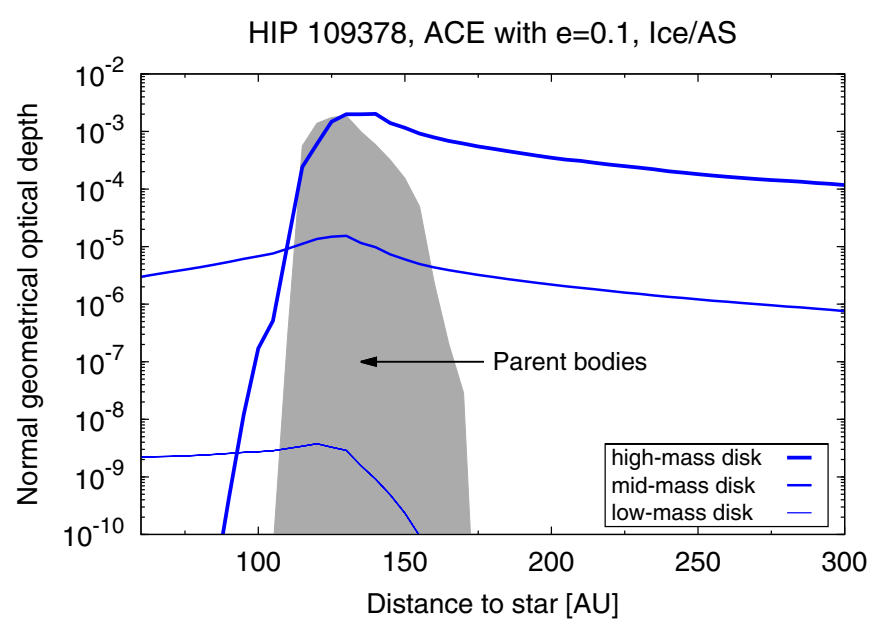

Figure 7. Radial profiles of the same disks as in Figure 6. The filled gray area shows the distribution of large, $1 \mathrm{~mm}$ sized grains (which we scaled vertically to the height of the other curves) to illustrate the position of the "parent ring." (A color version of this figure is available in the online journal.)

dominated, whereas the low-mass one is transport dominated. In the former case, the size distribution peaks at grains as small as several $\mu \mathrm{m}$, and the radial profile extends inward from the parent ring position only moderately. In the latter case, we observe the expected significant shift in the size of grains that dominate the cross section to several hundreds of $\mu \mathrm{m}$, and the radial profile reveals that transport efficiently fills the inner void of the disk with dust. The transition from the collision- to the transportdominated regime is found to occur around the dustiness level of the mid-mass disk. The size distribution in that disk still resembles that of the high-mass disk, but the inner gap in the disk is essentially filled with material, similar to the low-mass disk case.

These results bring up a major question. Is the optical depth of our cold disk candidates, exemplified by HIP 109378 here, low enough to put them into the transport-dominated regime? To answer this, we used SEDUCE to calculate the SEDs of the three modeled disks and compared them with the observed emission

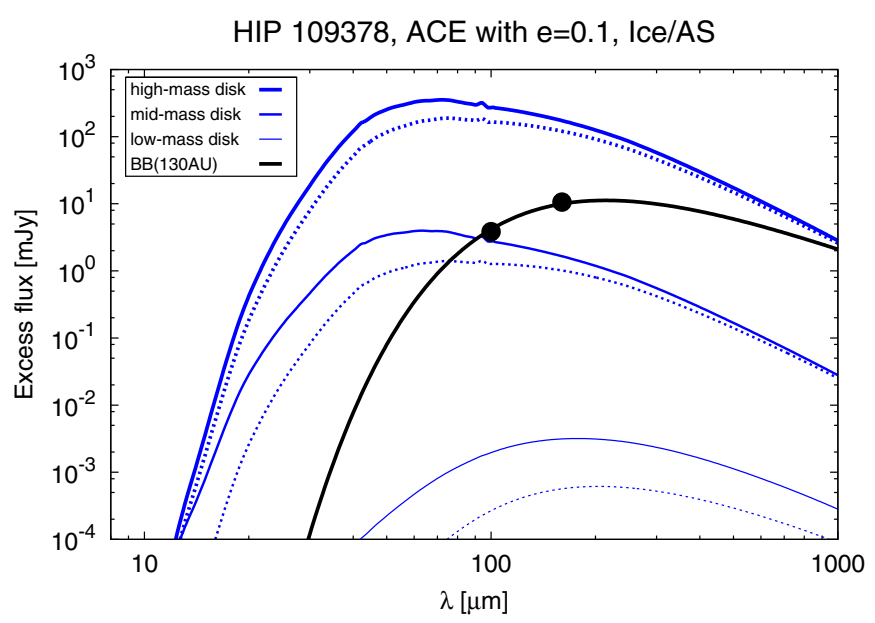

Figure 8. SEDs of the disks presented in Figures 6 and 7 (blue solid lines). The contributions from the "parent ring" (more exactly, from region between $120 \mathrm{AU}$ and $140 \mathrm{AU}$ ) are shown with dashed lines. The "blackbody" SED (thick solid line) and the observed excess emission of HIP 109378 (symbols; error bars are smaller than the data points) are plotted for comparison and are the same as in Figure 5 (top).

(A color version of this figure is available in the online journal.)

level in Figure 8 (solid lines). The answer is clearly negative. The optical depth of the disk of HIP 109378 (and the other cold disk candidates) lies between the high- and mid-mass cases. As a result, the dominant grains are still in the $\mu \mathrm{m}$ range, and the modeled SEDs of both the high-mass and mid-mass disks are too "warm." They would imply that the excess emission at $70 \mu \mathrm{m}$ is stronger than at $160 \mu \mathrm{m}$ (and that the excess emission at $70 \mu \mathrm{m}$ is extended). This is not observed, even for HIP 92043 and HIP 73100, which do reveal a $70 \mu \mathrm{m}$ excess.

Furthermore, even if the disk had an optical depth low enough to match the low-mass regime, this solution would have to be ruled out because of the unavoidable contribution of the warmer small grains drifting to the inner regions from the parent ring. This is clearly apparent from Figure 8 . The dashed lines plot the contributions to the overall emission made by the material in the parent ring region. Judging by the difference between the solid and dashed curves, we conclude that, indeed, the dust inside the ring leads to a noticeable increase of the fluxes and slightly shifts the peak of the SEDs toward shorter wavelengths. (Note that the contribution of the "halo" grains outside the ring is negligible. We have checked that, for instance, placing the outermost distance at $300 \mathrm{AU}$ instead of $200 \mathrm{AU}$ leaves the SEDs almost unchanged.)

\subsection{Disks with Low Dynamical Excitation}

Another possibility is to assume that dust-producing planetesimals have a low dynamical excitation which, however, is still high enough for collisions to be mostly destructive. In this case, low collision velocities between large grains, insusceptible to radiation pressure, would create an imbalance between the rates at which small grains are produced (low) and destroyed (high). As a result, the disk would be devoid of small particles (Thébault \& Wu 2008).

This scenario has been confirmed previously with detailed ACE simulations by Löhne et al. (2012), who modeled the Herschel/DUNES disk around HD 207129 (Marshall et al. 2011). Similar to our cold disk candidates, it has a solar-type central star and a radius of $\gtrsim 100 \mathrm{AU}$, but its fractional luminosity is appreciably higher and the emission is warmer. Löhne 


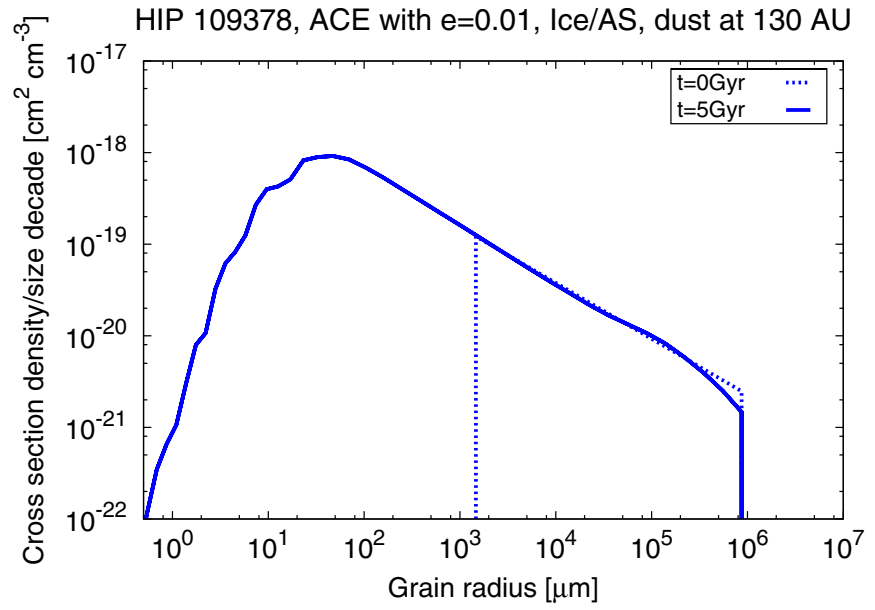

Figure 9. Simulated evolution of the size distribution in a disk with $e \sim 0.01$ macroscopic grains under the conditions of HIP 109378. Dashed line: assumed initial distribution; solid line: distribution after $5 \mathrm{Gyr}$ of collisional evolution. (A color version of this figure is available in the online journal.)

et al. (2012) have shown that a low dynamical excitation with $e \sim I \sim 0.03$ shifts the grain size at which the size distribution peaks from $\approx 0.5 \mu \mathrm{m}$ to $\approx 3-4 \mu \mathrm{m}$. However, to be able to explain the cold disks, the effect needs to be stronger and the dynamical excitation lower. Indeed, for the size distribution to peak at $\sim 100 \mu \mathrm{m}$ in the Thébault \& Wu (2008) scenario, we would need $e \sim 0.001$. Note that Thébault \& Wu (2008) made their simulations for A-type stars, for which the blowout size is larger than for FGK stars considered here, which would necessitate even lower eccentricities. The eccentricities $e \lesssim 0.001$ would correspond to relative velocities of $\lesssim 3 \mathrm{~m} \mathrm{~s}^{-1}$. Numerous laboratory experiments and microphysics simulations (see Blum \& Wurm 2008, for a review) tell us that at this level, the collisions are not necessarily destructive and the scenario of Thébault \& Wu (2008) in its proposed form may no longer be applicable.

To check more quantitatively what exactly happens at excitation levels lower than those previously simulated with ACE ( $e \sim I \sim 0.03$ ), and to what extent the effects predicted by Thébault \& Wu (2008) come into play, we now explore the $e \sim 0.01$ regime. We ran ACE for the disk around HIP 109378, and assumed a planetesimal ring centered at $130 \mathrm{AU}$, initially composed of large grains with initial radii between $1 \mathrm{~mm}$ and $\approx 1 \mathrm{~m}$ (with a differential size distribution slope of 3.7 ). Note that the maximum size was arbitrarily set to $\approx 1 \mathrm{~m}$; we could have taken any value below $\sim 30 \mathrm{~km}$ (see estimates in the end of Section 4.4), for which the largest planetesimals do not yet stir the disk to higher values of eccentricity and inclination than the ones we assumed in this simulation. The same applies to the minimum size: we made a separate test run with $s_{\min }=1 \mu \mathrm{m}$ instead of $1 \mathrm{~mm}$ and did not see any appreciable difference. As in Section 4.1, we took a homogeneous mixture of astrosilicate and water ice in equal volume fractions. The choice of the initial total disk mass is trickier, since we have to come up with the fluxes at the observed level, but we do not know in advance what the evolved size distribution will look like. With a few "guessand-try" attempts, the "right" disk mass was finally found to be $0.02 M_{\oplus}$. We used uniformly distributed eccentricities between 0.005 and 0.015 and inclinations of $\leqslant 0.01$, which would correspond to relative velocities of $\sim 30 \mathrm{~m} \mathrm{~s}^{-1}$. In this velocity regime, in contrast to that considered in Section 4.1, we enter the conditions probed by numerous direct laboratory impact
HIP 109378, ACE with $e=0.01$, Ice/AS, dust at $130 \mathrm{AU}$

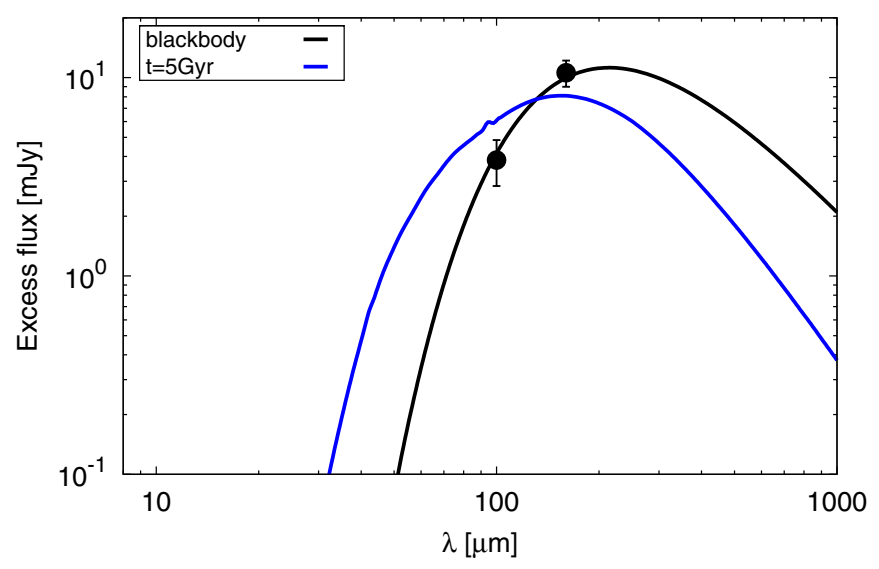

Figure 10. SEDs of the same disk as in Figure 11 (blue line). The "blackbody" SED (black line) and the observed excess emission of HIP 109378 (symbols with error bars which are smaller than the symbol size) are shown for comparison and are the same as in Figures 5(a) and (b).

(A color version of this figure is available in the online journal.)

experiments. Accordingly, we used a flat (index $q=2.0$ ) size distribution of fragments, as suggested by experiments (Güttler et al. 2010). The other parameters, including $Q_{D}^{\star}$, were the same as in the previous runs (see Section 4.1).

The evolution of the size distribution after 5 Gyr is shown in Figure 9. These results demonstrate that collisional production of small grains is still quite efficient. Although there occurs a shift of the dominating sizes toward large values, as predicted by Thébault \& Wu (2008), the effect is not strong enough. The dominating grains are only a few tens of micrometers in radius. The resulting SED shown in Figure 10, although coming closer to the available photometry points of HIP 109378, still appears too "warm."

\subsection{Unstirred Disks with "Jostling” Collisions}

We now explore the regime of very low dynamical excitations and very low collisional velocities. We start with simple analytic considerations. Since we are now interested in a disk at a very low dynamical excitation level, one may be wondering whether collisions in such a disk are frequent enough to play any role at all. Assuming grains in almost circular, non-inclined orbits, one might expect to get a system with very long collisional timescales, perhaps exceeding the system's age. However, this is not true. For a narrow disk with a radius $r$ composed of equalsized grains, the collisional timescale is of the order of (Backman \& Paresce 1993; Artymowicz \& Clampin 1997; Wyatt et al. 1999; Wyatt 2005)

$$
T_{\text {coll }}=\frac{P}{2 \pi \tau_{\perp}},
$$

where $P$ is the orbital period at $r$ and $\tau_{\perp}$ is the normal geometrical optical depth of the disk at that distance. Interestingly, Equation (3) does not depend on eccentricities and inclinations. This is because, in the first order in $e$ and $I, T_{\text {coll }}$ is proportional to the "effective interaction volume of particles" and inversely proportional to their relative velocity. Both, in turn, are proportional to the eccentricities and inclinations, so these cancel out (see, e.g., Krivov et al. 2007, their Equations (15)-(18)).

For each disk, the dust luminosity and the position of the SED maximum are known, at least roughly. These two quantities uniquely determine both the total cross section 
of material and the characteristic disk radius $r$ which, via Equation (3), immediately fix $T_{\text {coll }}$. Although real disks must have a distribution of grain sizes (making $T_{\text {coll }}$ size dependent) and may be radially extended (making $T_{\text {coll }}$ larger, since the same amount of emission would be reached at a lower $\tau_{\perp}$ ), Equation (3) allows a ball-park estimate. For a disk around a solar-type star with an optical depth of $\tau_{\perp}=10^{-6}$ and a radius of $r=100 \mathrm{AU}$, we have $T_{\text {coll }} \sim 200 \mathrm{Myr}$. Since the host stars of the cold disks have ages of Gyr (see Table 1), we conclude that the collisions cannot be ignored. This conclusion will be directly confirmed by collisional simulations described below.

Since the system will not be collisionless even at low dynamical excitations, we can only try to find a range of relative velocities that would allow macroscopic primordial grains ( $s \gtrsim 1 \mathrm{~mm}$ ) to survive, while at the same time not producing too many "unwanted" small grains. The velocities should not be too large, otherwise collisions will be too destructive, creating too many small fragments. They should not be too small either, because collisions at very low velocities will be $100 \%$ sticking, leading to a rapid loss of the net cross section of the material. In fact, the results of laboratory experiments and numerical simulations uncover a very complex view (see Blum \& Wurm 2008 and references therein). They show that the collisional outcome depends on impact velocity and impact angle, masses, materials, porosities, and hardnesses of the projectile and target, radius of curvature of the target surface, morphology, size ratios of the impactors, and other factors. The bouncing regime, which would be the most favorable for the cold disk scenario to work, typically occurs at sizes $1-10 \mathrm{~cm}$, or velocities from a few $\mathrm{cm} \mathrm{s}^{-1}$ to a few $\mathrm{m} \mathrm{s}^{-1}$. Note, however, that the behavior depends sensitively on the grain morphology (Poppe et al. 2000). As an example, spherical particles possess a rather well-defined threshold velocity around $1 \mathrm{~m} \mathrm{~s}^{-1}$, below which they always stick and above which they never do. However, irregularly shaped dust particles may stick even at impact velocities of several tens of meters per second in "hit-the-wall" collisions.

In what follows, we check whether the desired regime can be reached at relative velocities of a few $\mathrm{ms}^{-1}$. This level of dynamical excitation (relative velocities of a few meters per second, or $e \sim I \sim 0.001$ for our cold disks) roughly agrees with the one that can be expected for the solids at the beginning of their collisional evolution, i.e., shortly after the dispersal of the primordial gas. Indeed, that level should be largely determined by turbulent velocities of the gas phase. At radii of $100 \mathrm{AU}$, one expects random velocities in the $\mathrm{m} \mathrm{s}^{-1}$ range for the smallest grains that were strongly coupled with gas at the protoplanetary ( $\mathrm{T}$ Tau) phase and somewhat higher ones ( $\sim 0.1$ of the sound speed) for weakly coupled ones (R. Nelson 2012, private communication).

To see whether the amount of small dust can be suppressed to a sufficient extent in this very low velocity scenario, we again used ACE. The setup was the same as in Section 4.2, except that we decreased the dynamical excitation by one order of magnitude. Specifically, we assumed uniformly distributed eccentricities between 0.0005 and 0.0015 and inclinations of $\leqslant 0.001$, resulting in relative velocities of $\sim 3 \mathrm{~m} \mathrm{~s}^{-1}$. Besides, we made three runs with dissimilar material strength and grain stickiness. In one run ("ref"), we use the standard values of $Q_{D}^{\star}$ (see the text after Equation (2)). In a separate ("sticky") run, we kept the same $Q_{D}^{\star}$, but assumed that in every cratering or bouncing collision, a fraction of cratered mass equal to $1-E_{\text {imp }} / E_{\text {crit }}\left(m_{\mathrm{p}}, m_{\mathrm{t}}\right)$ remains stuck to the target instead of being ejected to space. Such an increased "stickiness"

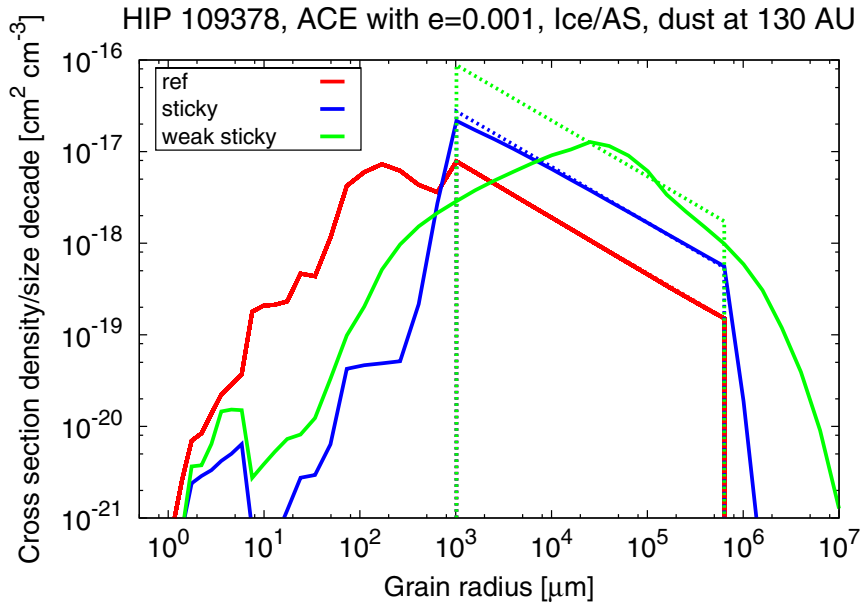

Figure 11. Simulated evolution of the size distribution of a dynamically cold belt of macroscopic grains under the conditions of HIP 109378. Dashed lines: assumed initial distributions; solid lines: distributions after $5 \mathrm{Gyr}$ of collisional evolution. The red, blue, and green lines correspond to the "ref," "weak," and "weak sticky" runs as described in the text.

(A color version of this figure is available in the online journal.)

was recently found experimentally for low-temperature ice by Windmark et al. (2012). Finally, in the "weak sticky" run, we assumed the same prescription of stickiness, but reduced $Q_{D}^{\star}$ by two orders of magnitude, as reported by Beitz et al. (2011). The total masses of the three disks, needed to produce the dust emission at the observed level, were again determined with an iterative "guess-and-try" procedure to be $0.07 M_{\oplus}$ ("ref"), $0.25 M_{\oplus}$ ("sticky"), and $0.8 M_{\oplus}$ ("weak sticky").

For the impact velocities and impactor sizes in question, the runs show a rather complex mixture of outcomes, including disruption, cratering and bouncing with mass transfer, and agglomeration in comparable fractions. The evolution of the size distribution over $5 \mathrm{Gyr}$ for all three runs is shown in Figure 11. Bumps in the distributions at smaller sizes are numerical artifacts, coming largely from a limited resolution of the phase-space grid. These results demonstrate both the accretional growth of solids to sizes above $1 \mathrm{~cm}$ (particularly significant in the "weak sticky" run) and a moderate amount of collisional fragments in the submillimeter range. The latter, however, are still large enough $(\sim 100 \mu \mathrm{m})$ to stay sufficiently cold. Indeed, the resulting SEDs, shown in Figure 12, are all close to the "blackbody SED." They are consistent at the $1 \sigma$ level with the available photometry points of HIP 109378.

We stress that the setup of these simulations is by far not unique, and thus the agreement with the data should not be overinterpreted. The purpose of these simulations was solely to demonstrate that the "macroscopic belt" scenario is conceivable and is potentially able to explain the observations.

\subsection{Constraints on Sizes}

We now try to paint a more general view. $s_{\min }$ and $s_{\max }$ indicate the "effective" minimum and maximum radius of the disk solids-i.e., the range of sizes beyond which the amount of particles is too small to affect the dynamical evolution and thermal emission of the disk. The largest objects have to be large enough to survive against various loss processes over the systems' ages which, in case of our cold disk candidates, are in the Gyr range. One of these loss mechanisms is P-R drag (further mechanisms are discussed in Section 5). Assuming 
HIP 109378, ACE with e=0.001, Ice/AS, dust at $130 \mathrm{AU}$

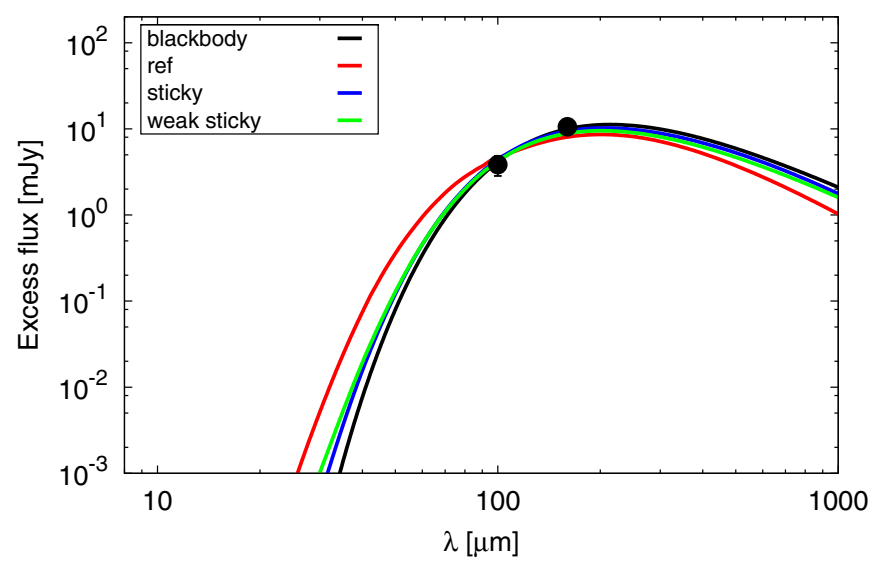

Figure 12. SEDs of the dynamically cold belt of macroscopic grains (red, blue, and green lines correspond to those in Figure 11). The "blackbody" SED (black line) and the observed excess emission of HIP 109378 (symbols with error bars) are shown for comparison and are the same as in Figures 5(a) and (b).

(A color version of this figure is available in the online journal.)

a unit radiation pressure efficiency, the associated timescale is given by

$$
T_{\mathrm{PR}} \sim 7\left(\frac{L_{\odot}}{L_{\star}}\right)\left(\frac{r}{100 \mathrm{AU}}\right)\left(\frac{\rho}{3 \mathrm{~g} \mathrm{~cm}^{-3}}\right)\left(\frac{s}{1 \mathrm{~mm}}\right) \mathrm{Gyr},
$$

where $\rho$ is the bulk density of dust. We conclude that $s_{\max } \gtrsim$ $1 \mathrm{~mm}$ would satisfy the P-R drag-induced condition.

The lower limit on $s_{\min }$ from the "coolness" of the SED $(\gtrsim 100 \mu \mathrm{m})$ and the lower limit on $s_{\max }$ from the P-R drag condition ( $\gtrsim 1 \mathrm{~mm}$ ) are not the only constraints that we can place. The upper limit on $s_{\max }$ can be estimated from the requirement that typical random velocities of grains $v_{\text {rel }}$ do not exceed a few tens of $\mathrm{m} \mathrm{s}^{-1}$. These random velocities cannot be lower than the relative velocities, to which grains are stirred by the biggest bodies embedded in the disk. The latter are approximately equal to the escape velocities from the surface of the largest planetesimals with radius $s_{\max }$ :

$$
v_{\text {rel }}>\sqrt{8 / 3 \pi G \rho} s_{\max } .
$$

For the bulk density $\rho \sim 2 \mathrm{~g} \mathrm{~cm}^{-3}$, this leads to a simple result: $s_{\max }<v_{\text {rel }}$, where $s_{\max }$ is in $\mathrm{km}$ and $v_{\text {rel }}$ in $\mathrm{m} \mathrm{s}^{-1}$. For instance, $v_{\text {rel }}$ of $3 \mathrm{~m} \mathrm{~s}^{-1}$ implies $s_{\max }<3 \mathrm{~km}$. For disks of a $\sim 100 \mathrm{AU}$ radius around a solar-type star, this sets the largest possible size of objects in the disk to a few kilometers.

Another requirement is that the total mass of the disk $M_{d}$, which is also determined by the largest objects, should not become unrealistically large. Assuming that the disk is composed of solids with sizes from $s_{\min }$ to $s_{\max }$ with the size distribution $\propto s^{-q}$, where $3<q<4$ and we take $q=3.5$ for simplicity, it is easy to derive the relation between $M_{d}$, the fractional luminosity $f_{d}$, as well as $s_{\min }$ and $s_{\max }: M_{d}=$ const . $f_{d} \cdot \sqrt{s_{\min } s_{\max }}$. The prefactor can be directly estimated from the simulations described in Section 4.3. For the HIP 109378 disk in the "sticky" run, in which the size distribution can roughly be approximated by a power law with $s_{\min } \approx 1 \mathrm{~mm}$, $s_{\max } \approx 1 \mathrm{~m}$, and $q \approx 3.5$, we obtained $f_{d} \approx 5 \times 10^{-6}$ with the disk mass of $M_{d} \approx 0.25 M_{\oplus}$. This gives

$$
M_{d} \approx 1.3 f_{d} \sqrt{s_{\min } s_{\max }},
$$

where $M_{d}$ is in the Earth masses and $s_{\min }$ and $s_{\max }$ in micrometers. Total disk masses exceeding $\sim 10 M_{\oplus}$ would probably be unrealistic. This is because the initial disk masses at protoplanetary stage are typically comparable with the minimum mass solar nebula (MMSN) mass, $0.01 M_{\odot}$, albeit with a large scatter (see Williams \& Cieza 2011, for a review of submillimeter observations) and, applying a standard 100:1 gas to mass ratio, are expected to contain $10-100 M_{\oplus}$ of solids. Furthermore, the mass of the cold disks is probably much less than that because it only contains a fraction of material that survived on the periphery of

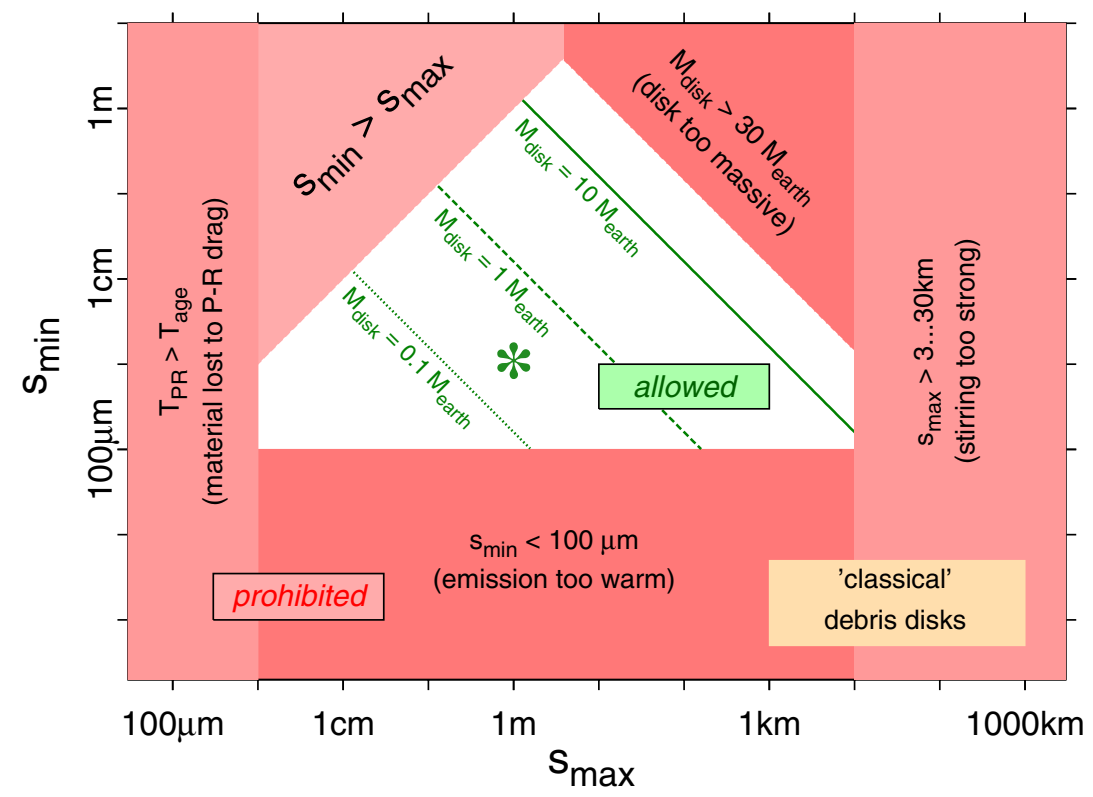

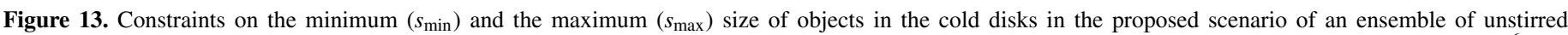

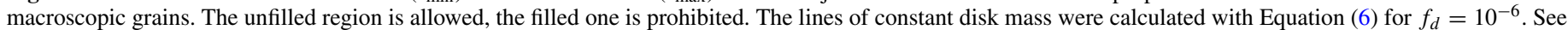

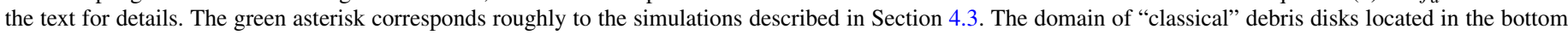
right corner is shown for comparison.

(A color version of this figure is available in the online journal.) 
the systems. For $s_{\min } \lesssim 1 \mathrm{~mm}$, this implies a constraint on $s_{\max }$ nearly as stringent as the "no stirring" constraint, Equation (5): $s_{\max } \lesssim 10 \mathrm{~km}$.

Figure 13 summarizes all these constraints, depicting the "allowed" region in the $s_{\min }-s_{\max }$ plane. It suggests that the observations are consistent with a hypothesis of an unstirred belt of macroscopic solids, whose sizes may lie somewhere in the millimeter to kilometer range.

\section{CONCLUSIONS AND DISCUSSION}

\subsection{Galaxies or Disks?}

In this paper, we consider six "cold debris disk" candidates identified by the Herschel OTKP DUNES. Since these observations are at risk of being contaminated by chance alignments of extragalactic sources, we cannot definitely rule out a possibility that any of these sources could be unrelated objects rather than true circumstellar disks. However, our catalog search for possible X-ray sources and their optical counterparts in the fields around the optical positions of the six stars has not identified any sources that could be associated with contaminating galaxies. Besides, a conservative estimate based on the density of galaxies in the DUNES fields leads to the probability that some of the candidates are real disks (and thus the "cold debris disk" phenomenon as such is real) of $\approx 99 \%$.

Final answers can be found by repeating the observations of the candidates with another instrument, ideally at more than one wavelength and in more than one epoch, in order to reveal the proper motion (or the absence thereof) of the sources of emission. For this purpose, one does not need to resolve the disk. Such observations will certainly be possible, for instance with ALMA. For point sources, $50 \mu \mathrm{Jy}$ detections $(1 \mathrm{hr}, 5 \sigma)$ at $1.3 \mathrm{~mm}$ (band 6 ) are possible, and this would be more than sufficient to detect the cold disk candidates. A successful ALMA detection after a few years might help to rule out background contamination via positional shifts of the source compared to field objects (which we have from PACS/160 $\mu \mathrm{m}$ or SPIRE/ $250 \mu \mathrm{m}$ ). If there is still an object at the old (Herschel) stellar position, then it is likely a contaminating galaxy. The only caveat to this is that ALMA's (sub)millimeter sky will probably be quite different from the Herschel's far-IR sky-as it actually is even among the three PACS bands. Thus, it would be better to observe the fields with ALMA at two epochs, in order to approach the problem under the same sky confusion background.

Beside ALMA, detection with CCAT (Sebring et al. 2006) ${ }^{27}$ and SPICA/SAFARI (Goicoechea \& Nakagawa 2011) should be possible. The sensitivity (point-source, $1 \mathrm{hr}$ integration, $5 \sigma$ ) of CCAT at its primary wavelengths of $350 \mu \mathrm{m}$ and $450 \mu \mathrm{m}$ should be about $1 \mathrm{mJy}$, and that of SPICA/SAFARI in the $100-200 \mu \mathrm{m}$ range as high as $30 \mu \mathrm{Jy}$. Besides, both instruments will be capable of performing high-resolution spectroscopy. This, especially in the case of SPICA/SAFARI with its excellent sensitivity, might allow another method to establish the nature of cold emission - by detecting or ruling out characteristic spectral features that might be typical of background galaxies.

Obviously, the most advantageous approach would be resolved observations of the candidates. Due to the higher angular resolution, the probability of contamination in general would be lower. The time span between two observations sufficient to confirm or rule out the common proper motion would be much shorter. Last but not least, a detection of a ring-like structure

\footnotetext{
27 See also http://www.ccatobservatory.org.
}

around the star (which might also be detected, depending on the setup and integration time) would be undeniably the best proof that we are dealing with a disk.

Which instrument has-or will have-the capability to sufficiently resolve the sources? SPICA, with its $3.2 \mathrm{~m}$ mirror, would have a resolving power comparable to that of Herschel, and thus would not offer any advantages. Using CCAT could be more promising, as its $25 \mathrm{~m}$ mirror would provide a resolution of 2.9 and 3.7 at $350 \mu \mathrm{m}$ and $450 \mu \mathrm{m}$, respectively, which is a factor of 3-4 better than that of PACS at $160 \mu \mathrm{m}$. However, the most promising instrument would be ALMA. The expected total excess flux from the cold disk candidates is $\sim 1 \mathrm{mJy}$ at $1.3 \mathrm{~mm}$, which would be reachable with a beam of $\approx 1^{\prime \prime}$. Given the point-source sensitivity quoted above, the disk can be distributed over 20 beams to be detected. Assuming an angular resolution of $1^{\prime \prime}$ and a face-on disk (worst case), the dust can be distributed over a ring of $\lesssim 3^{\prime \prime}$ in radius to be still detected at $5 \sigma$. For the edge-on disk (best case), a radius of $\lesssim 10^{\prime \prime}$ would suffice. The range $3^{\prime \prime}-10^{\prime \prime}$ is approximately what we expect for the cold disks from the Herschel data.

\subsection{If Disks, Are They Dynamically Cold?}

For true circumstellar disks, and assuming that the gravitational perturbers (planets, substellar or stellar companions) are absent, the proposed scenario of dynamically cold (i.e., unstirred) disks would imply a rotationally symmetric, ring-like confinement of material and a lack of any observable offsets or clumps. If a perturber is present, then the disk could still be dynamically cold if the disk particles shared the same forced eccentricities induced by the perturber, but had proper eccentricities close to zero, keeping the relative velocities at a very low level. It may be possible if the particles' eccentricities were initially close to the forced eccentricity from the perturber, and remained there for the disk's lifetime. This may be achievable if the particles' eccentricities were damped to the forced eccentricity when a gas disk was still present (Thébault 2012), or if collisions between particles occur frequently enough and at low enough velocity to damp eccentricities in the collisions without destroying the particles. The disk would be elliptic, similar to the Fomalhaut ring (Kalas et al. 2008; Chiang et al. 2009). In this case, there would be an offset, and possibly a pericenter glow (Wyatt et al. 1999).

Verifying these possibilities appears to be a more difficult task than confirming the circumstellar origin of the emission because it requires the disk to be well resolved. However, as discussed in Section 5.1, sufficiently well resolved observations with ALMA seem possible.

\subsection{Possible Implications for Planetesimal Formation?}

Assuming that the observed emission is indeed related to the stars, we concluded that the emitting material should have nearly a blackbody temperature. We then examined possibilities to explain why the emitting material in these disks is thermally cold. We argued that the cold disks should be composed of weakly stirred or unstirred primordial solids with radii in the range from millimeters to about $10 \mathrm{~km}$. Tighter constraints on sizes are difficult to pose, but solids larger than $\sim 1 \mathrm{~mm}$ are needed for the material to survive against P-R drag, while objects larger than $\sim 10 \mathrm{~km}$ must be absent, since these would stir the disk out of its "cold" state. Neither is it possible to put more stringent constraints on the degree of dynamical excitation in the disks. We showed that the maximum eccentricities and inclinations should not exceed $\sim 0.01$, since the amount of small 
dusty debris would otherwise contradict the observational data. However, these might easily be as low as $\sim 0.001$, corresponding to relative velocities of a few $\mathrm{m} \mathrm{s}^{-1}$. Our simulations suggest that a system with $e \sim I \sim 0.001-0.01$ would experience gentle collisions that only involve a moderate production of small dusty debris and a moderate amount of (further) accretional growth, and could survive around a star for gigayears. A principal possibility for systems of this type to exist was recently pointed out by Heng \& Tremaine (2010), and Saturn's rings in the solar system readily provide an example of such disks, albeit on a much smaller spatial scale (Esposito 2002).

We now compare our findings with models of planetesimal growth. At early phases, all these models have to overcome various hurdles. One is a rapid loss of material due to radial drift at some sizes (e.g., Weidenschilling 1980) and another is a switch from the agglomerational to fragmentational regime at growing sizes (e.g., Blum \& Wurm 2008 and references therein). Under the MMSN conditions at $\sim 1 \mathrm{AU}$, both are expected to happen at sizes of about $1 \mathrm{~m}$, and thus are often referred to as the "meter barrier." However, in the outer parts of the systems (at $\sim 100 \mathrm{AU}$ ), drift is fastest at millimeters or centimeters rather than decimeters or meters (Brauer et al. 2007). Various ways have been suggested to circumvent both the drift and fragmentation hurdles. The drift problem may not exist in turbulent disks, and models with more and more realistic physics involved may also help to eliminate or at least mitigate the fragmentation problem (e.g., Brauer et al. 2008; Zsom et al. 2010, 2011). Alternative pathways for a rapid unimpeded growth have also been proposed that invoke collective dust phenomena, such as capture of solids in pressure maxima of the gas disk (Johansen et al. 2006), possibly enhanced by streaming instability (Johansen et al. 2007), both with subsequent local gravitational clumping of material (see Chiang $\&$ Youdin 2010, for a review). Whatever path the system takes-if kilometer or larger sizes have been reached-further growth of planetesimals is expected in gravity-assisted pairwise collisions (e.g., Goldreich et al. 2004).

All models naturally predict the planetesimal growth to strongly depend on the distance from the star. At any given age, the largest objects are smaller farther out, and conversely, it takes longer for the planetesimals to grow to a given size farther out from the central star. Effectively, the growth should stall at large distances from the star, where the nebula has a very low density. Furthermore, protoplanetary disks have finite sizes, being truncated by internal physical processes or external influences such as stellar encounters. These sizes are probed by observations (see Williams \& Cieza 2011, for a review). (Sub)millimeter data suggest exponentially tapered outer edges of disks to lie between a few tens and a few hundreds of AU, with radii of $\sim 100$ AU being typical (e.g., Andrews et al. 2009, 2010). A similar range of radii, $\sim 50$ to $\sim 200 \mathrm{AU}$, comes from direct measurements of silhouette proplyds (Vicente \& Alves 2005). With their estimated radii of $\sim 50-150 \mathrm{AU}$, the cold disks may thus simply trace the outer edges of the original planetesimal disks, namely, the maximum distance up to which planetesimals could form.

More specific conclusions are extremely difficult to arrive at. As explained above, we cannot predict whether solids in the cold disks are millimeters or kilometers in sizes. We cannot even be sure whether they entered the gravity-driven growth regime. Previous observations of protoplanetary disks do tell us that dust growth on the periphery of the systems advances at least to millimeters (e.g., Wilner et al. 2005; Rodmann et al. 2006), but they do not probe larger sizes. The model predictions for the planetesimal formation timescales and final sizes are extremely uncertain too. Kenyon \& Bromley (2008), for instance, assumed initial planetesimal sizes of $1 \mathrm{~m}-1 \mathrm{~km}$ and modeled planetesimal growth in the 30-150 AU range around 1-3 $M_{\odot}$ stars in a set of possible nebulae with $(0.3-3) \times \mathrm{MMSN}$ density profiles. They found that after $\sim 1 \mathrm{Gyr}$ of evolution, the largest planetesimals in an MMSN disk reach sizes of $\sim 100 \mathrm{~km}$ even at $r \sim 150 \mathrm{AU}$. This might conflict with our conclusion that the planetesimal growth in cold disks must have stopped before these "cometary" or "asteroidal" sizes have been reached. The possible controversy can, however, easily be mitigated by varying the model assumptions, for instance, the density profile of the original disk. Assuming $0.3 \times \mathrm{MMSN}$ reduces the maximum sizes to $\sim 30 \mathrm{~km}$, close to what is needed. Alternative models of rapid planetesimal formation that invoke clumping of material in turbulent disks are not certain in their predictions either. For instance, Johansen et al. (2012) find the final sizes of Kuiper objects formed in this way to be $150-730 \mathrm{~km}$, but it is easy to imagine that the same mechanisms at distances of $\sim 100 \mathrm{AU}$ and/or under different model assumptions could halt at smaller sizes.

\subsection{Planets in the Cold Disk Systems?}

Cold disks must have large inner voids, as inferred from the absence of emission at $\lesssim 100 \mu \mathrm{m}$. In the inner parts of the "cold disk" systems, the planetesimal formation is likely to have advanced further, ending up with the formation of planets (J. P. Marshall et al., in preparation). Indeed, one of the cold disks, HIP 109378, was reported to host a radial velocity planet (Marcy et al. 1999).

Whether planets are present farther out from the stars in the inner gaps of the cold disks is unclear. If they were, then they would naturally account for these inner voids. However, as discussed in Section 5.2, the presence of planets would have to be compromised with the suggested dynamically cold state of the disks by requiring low proper eccentricities of the disk particles from their formation stage or from the subsequent collisional damping. Conversely, if the planets were absent, the disks could preserve their dynamically cold state easily. However, this would raise the question of what, if not planets, has created the inner voids. A few mechanisms that do not necessarily involve planets appear feasible. These may include, for instance, dust drag triggered by UV-switch (e.g., Alexander \& Armitage 2007) and collisional depletion of the inner region of an initially extended planetesimal disk (e.g., Wyatt et al. 2012).

Interestingly, one of the six stars, HIP 92043, shows a $K$-band excess of around $1 \%$, which has been interpreted as stemming from a hot exozodiacal cloud (Absil et al. 2013). Such exozodis have previously been detected with CHARA/FLUOR around Vega, Fomalhaut, $\tau$ Cet, and some other stars (see Absil \& Mawet 2010, for a recent review). The origin of these exozodis is as yet unclear; these may or may not be related to the outer disks (Bonsor et al. 2012).

\subsection{Could Disks Survive and Remain Cold for Gigayears?}

Most of the above discussion is about the very early stages of planetesimal formation. From solar system (Morbidelli 2010) and exoplanet system studies (Booth et al. 2009; Raymond et al. 2011, 2012), we know that a planetary system may undergo violent dynamical rearrangements that might smear 
out or substantially alter the architecture left immediately after the completion of the protoplanetary phase. Even if the systems evolve in a smooth way, cold belts on the periphery might be threatened by a variety of effects over Gyr timescales. These include possible interactions of disks with the ISM, especially during passages through clouds, and galactic tidal forces.

For HIP 171, which is a binary star, the cold disk-if it is real-would be circumbinary. Since the binary has a substantial eccentricity (0.38), it is not clear whether the outer disk could have remained unstirred over the system's age, even though the semimajor axis ratio is large (Mustill \& Wyatt 2009). Simulations for a disk of particles with a radius of $R_{\text {disk }}=60 \mathrm{AU}$ (Table 2 ) initially in orbits with eccentricities between 0 and 0.1 showed that after 1 Gyr the eccentricity dispersion of the particles, induced by the binary, is of the order of 0.03-0.04 (A. J. Mustill et al., in preparation). This is larger than what is required by the dynamically cold scenario. Still, there exists a possibility to keep the eccentricity oscillations low if the particles' eccentricities are initially close to the forced eccentricity from the binary, and remain there for the disk's lifetime-see a discussion in Section 5.2.

\subsection{Concluding Remarks}

We conclude by noting that, regardless of whether all of the "cold disks" are true disks or some are unrelated background sources, the theoretical analysis of collisional evolution in different dynamical regimes presented in Section 4 of this paper may be of generic interest. This is because we try to find out how debris disks could operate at very low optical depths and at a low level of stirring, as can particularly be expected for disks with large radii. Both domains comfortably lie in the discovery space of the far-IR, submillimeter, and radio facilities that are starting to operate (such as SCUBA-2 and ALMA) or are being developed (e.g., SPICA). Thus, our analysis might provide useful guidelines for interpretation of the data that are expected to come.

\section{SUMMARY}

1. In this paper, we consider six "cold debris disk" candidates identified by the Herschel OTKP DUNES and argue that at a high level of confidence, most of these candidates represent true circumstellar disks.

2. For true circumstellar disks, the available data suggest that the dominant size of the grain cross section is larger than $\sim 100 \mu \mathrm{m}$ and that the smaller grains are strongly underabundant. This contrasts with all of the debris disks observed previously, where observations and models both reveal dominant sizes to lie in the micrometer range.

3. A plausible explanation for the dearth of small grains is the unstirred disks of solids grown on the periphery of systems during the protoplanetary phase. We show that to explain the data, they should comprise solids larger than millimeters, but smaller than a few kilometers in size. This would imply that planetesimal accretion, at the least in outer regions of the systems, has stopped before "cometary" or "asteroidal" sizes were reached.

We are grateful to the referee for useful comments that greatly helped to improve the manuscript. A.V.K. and T.L. thank Jürgen Blum and Carsten Güttler for their explanations on collisional outcomes and Ludwig Trepl for discussions on the ROSAT data. The work of A.V.K., T.L., and S.W. was partly funded by the Deutsche Forschungsgemeinschaft (grants Kr 2164/10-1, Lo 1715/1-1, and Wo 857/7-1). C.E., J.P.M., and B.M. were partly supported by Spanish grant AYA 2011-26202. J.-C.A. and S.E. acknowledge financial support of the CNES-PNP. S.E. also thanks the French National Research Agency (ANR) for financial support through contract ANR-2010 BLAN-0505-01 (EXOZODI). A.B. was co-funded under the Marie Curie Actions of the European Commission (FP7-COFUND).

\section{REFERENCES}

Absil, O., Defrère, D., Coudé du Foresto, V., et al. 2013, A\&A, in press

Absil, O., \& Mawet, D. 2010, A\&ARv, 18, 317

Alexander, R. D., \& Armitage, P. J. 2007, MNRAS, 375, 500

Anderson, E., \& Francis, C. 2011, yCat, 5137, 0

Anderson, S. F., Margon, B., Voges, W., et al. 2007, AJ, 133, 313

Andrews, S. M., Wilner, D. J., Hughes, A. M., Qi, C., \& Dullemond, C. P. 2009, ApJ, 700, 1502

Andrews, S. M., Wilner, D. J., Hughes, A. M., Qi, C., \& Dullemond, C. P. 2010, ApJ, 723, 1241

Artymowicz, P., \& Clampin, M. 1997, ApJ, 490, 863

Bach, K., Lee, J., Demarque, P., \& Kim, Y.-C. 2009, ApJ, 703, 362

Backman, D., \& Paresce, F. 1993, in Protostars and Planets III, ed. E. H. Levy \& J. I. Lunine (Tucson, AZ: Univ. Arizona Press), 1253

Barucci, M. A., Alvarez-Candal, A., Merlin, F., et al. 2011, Icar, 214, 297

Beitz, E., Güttler, C., Blum, J., et al. 2011, ApJ, 736, 34

Benz, W., \& Asphaug, E. 1999, Icar, 142, 5

Berta, S., Magnelli, B., Nordon, R., et al. 2011, A\&A, 532, A49

Blum, J., \& Wurm, G. 2008, ARA\&A, 46, 21

Bohren, C. F., \& Huffman, D. R. 1983, Absorption and Scattering of Light by Small Particles (New York: Wiley)

Bonsor, A., Augereau, J.-C., \& Thebault, P. 2012, A\&A, 548, A104

Booth, M., Wyatt, M. C., Morbidelli, A., Moro-Martín, A., \& Levison, H. F. 2009, MNRAS, 399, 385

Boyajian, T. S., von Braun, K., van Belle, G., et al. 2012, ApJ, 757, 112

Brauer, F., Dullemond, C. P., \& Henning, T. 2008, A\&A, 480, 859

Brauer, F., Dullemond, C. P., Johansen, A., et al. 2007, A\&A, 469, 1169

Brott, I., \& Hauschildt, P. H. 2005, in The Three-Dimensional Universe with Gaia, ed. C. Turon, K. S. O'Flaherty, \& M. A. C. Perryman (ESA SP-576; Noordwijk: ESA), 565

Chiang, E., Kite, E., Kalas, P., Graham, J. R., \& Clampin, M. 2009, ApJ, 693,734

Chiang, E., \& Youdin, A. N. 2010, ARE\&PS, 38, 493

Condon, J. J., Anderson, M. L., \& Helou, G. 1991, ApJ, 376, 95

Condon, J. J., Cotton, W. D., Greisen, E. W., et al. 1998, AJ, 115, 1693

Cutri, R. M., Skrutskie, M. F., van Dyk, S., et al. 2003, 2MASS All Sky Catalog of Point Sources

Davis, D. R., Chapman, C. R., Weidenschilling, S. J., \& Greenberg, R. 1985, Icar, 62,30

del Burgo, C., Laureijs, R. J., Ábrahám, P., \& Kiss, C. 2003, MNRAS, 346, 403

Draine, B. T. 2003a, ARA\&A, 41, 241

Draine, B. T. 2003b, ApJ, 598, 1017

Draine, B. T. 2003c, ApJ, 598, 1026

Durda, D. D., \& Dermott, S. F. 1997, Icar, 130, 140

Durda, D. D., Greenberg, R., \& Jedicke, R. 1998, Icar, 135, 431

Eggenberger, A., Udry, S., Chauvin, G., et al. 2007, A\&A, 474, 273

Eiroa, C., Fedele, D., Maldonado, J., et al. 2010, A\&A, 518, L131

Eiroa, C., Marshall, J. P., Mora, A., Krivov, A. V., et al. 2011, A\&A, 536, L4

Eiroa, C., Marshall, J. P., Mora, A., et al. 2013, A\&A, in press (arXiv:1305.0155)

Esposito, L. W. 2002, RPPh, 65, 1741

Fabian, D., Henning, T., Jäger, C., et al. 2001, A\&A, 378, 228

Flesch, E., \& Hardcastle, M. J. 2004, A\&A, 427, 387

Goicoechea, J. R., \& Nakagawa, T. 2011, in EAS Publications Series, Vol. 52, Conditions and Impact of Star Formation, ed. M. Röllig, R. Simon, V. Ossenkopf, \& J. Stutzki (Cambridge: Cambridge Univ. Press), 253 Goldreich, P., Lithwick, Y., \& Sari, R. 2004, ARA\&A, 42, 549

Griffin, M. J., Abergel, A., Abreu, A., et al. 2010, A\&A, 518, L3

Güdel, M. 1992, A\&A, 264, L31

Güttler, C., Blum, J., Zsom, A., Ormel, C. W., \& Dullemond, C. P. 2010, A\&A, 513, A56

Hauck, B., \& Mermilliod, M. 1998, A\&AS, 129, 431

Heng, K., \& Tremaine, S. 2010, MNRAS, 401, 867

Holland, W. S., Greaves, J. S., Zuckerman, B., et al. 1998, Natur, 392, 788

Holsapple, K. A. 1994, P\&SS, 42, 1067

Hughes, A. M., Wilner, D. J., Mason, B., et al. 2012, ApJ, 750, 82 
Johansen, A., Klahr, H., \& Henning, T. 2006, ApJ, 636, 1121 Johansen, A., Oishi, J. S., Low, M.-M. M., et al. 2007, Natur, 448, 1022 Johansen, A., Youdin, A. N., \& Lithwick, Y. 2012, A\&A, 537, A125

Kalas, P., Graham, J. R., Chiang, E., et al. 2008, Sci, 322, 1345

Kenyon, S. J., \& Bromley, B. C. 2004, AJ, 128, 1916

Kenyon, S. J., \& Bromley, B. C. 2008, ApJS, 179, 451

Kimura, H., Ishimoto, H., \& Mukai, T. 1997, A\&A, 326, 263

Koerner, D. W., Kim, S., Trilling, D. E., et al. 2010, ApJL, 710, L26

Krivov, A. V. 2010, RAA, 10, 383

Krivov, A. V., Löhne, T., \& Sremčević, M. 2006, A\&A, 455, 509

Krivov, A. V., Mann, I., \& Krivova, N. A. 2000, A\&A, 362, 1127

Krivov, A. V., Müller, S., Löhne, T., \& Mutschke, H. 2008, ApJ, 687, 608

Krivov, A. V., Queck, M., Löhne, T., \& Sremčević, M. 2007, A\&A, 462, 199

Krivov, A. V., Sremčević, M., \& Spahn, F. 2005, Icar, 174, 105

Kuchner, M. J., \& Stark, C. C. 2010, AJ, 140, 1007

Lebreton, J., Augereau, J.-C., Thi, W.-F., et al. 2012, A\&A, 539, A17

Lellouch, E., Kiss, C., Santos-Sanz, P., et al. 2010, A\&A, 518, L147

Li, A., \& Greenberg, J. M. 1998, A\&A, 331, 291

Lim, T. L., Stansberry, J., Müller, T. G., et al. 2010, A\&A, 518, L148

Löhne, T., Augereau, J.-C., Ertel, S., et al. 2012, A\&A, 537, A110

Löhne, T., Krivov, A. V., \& Rodmann, J. 2008, ApJ, 673, 1123

Love, S. G., Hörz, F., \& Brownlee, D. E. 1993, Icar, 105, 216

Marcy, G. W., Butler, R. P., Vogt, S. S., Fischer, D., \& Liu, M. C. 1999, ApJ, 520, 239

Marshall, J. P., Löhne, T., Montesinos, B., et al. 2011, A\&A, 529, A117

Morbidelli, A. 2010, CRPhy, 11, 651

Mukai, T., Ishimoto, H., Kozasa, T., Blum, J., \& Greenberg, J. M. 1992, A\&A, 262,315

Müller, S., Löhne, T., \& Krivov, A. V. 2010, ApJ, 708, 1728

Mustill, A. J., \& Wyatt, M. C. 2009, MNRAS, 399, 1403

O’Brien, D. P., \& Greenberg, R. 2003, Icar, 164, 334

Ott, S. 2010, in ASP Conf. Ser. 434, Astronomical Data Analysis Software and Systems XIX, ed. Y. Mizumoto, K.-I. Morita, \& M. Ohishi (San Francisco, CA: ASP), 139

Pál, A., Kiss, C., Müller, T. G., et al. 2012, A\&A, 541, L6

Paolicchi, P., Verlicchi, A., \& Cellino, A. 1996, Icar, 121, 126

Pilbratt, G. L., Riedinger, J. R., Passvogel, T., et al. 2010, A\&A, 518, L1

Poglitsch, A., Waelkens, C., Geis, N., et al. 2010, A\&A, 518, L2

Poppe, T., Blum, J., \& Henning, T. 2000, ApJ, 533, 454

Preibisch, T., Ossenkopf, V., Yorke, H. W., \& Henning, T. 1993, A\&A, 279, 577

Raymond, S. N., Armitage, P. J., Moro-Martín, A., et al. 2011, A\&A, $530, \mathrm{~A} 62$
Raymond, S. N., Armitage, P. J., Moro-Martin, A., et al. 2012, A\&A, 541, A11

Rodmann, J., Henning, T., Chandler, C. J., Mundy, L. G., \& Wilner, D. J. 2006, A\&A, 446, 211

Santos-Sanz, P., Lellouch, E., Fornasier, S., et al. 2012, A\&A, 541, A92

Schmitt, J. H. M. M. 1997, A\&A, 318, 215

Sebring, T. A., Giovanelli, R., Radford, S., \& Zmuidzinas, J. 2006, Proc. SPIE, $6267,62672 \mathrm{C}$

Shimaki, Y., \& Arakawa, M. 2012, Icar, 218, 737

Sibthorpe, B., Ivison, R. J., Massey, R. J., et al. 2013, MNRAS, 428, 6

Sibthorpe, B., Vandenbussche, B., Greaves, J. S., et al. 2010, A\&A, 518, L130

Skrutskie, M. F., Cutri, R. M., Stiening, R., et al. 2006, AJ, 131, 1163

Stewart, S. T., \& Leinhardt, Z. M. 2009, ApJL, 691, L133

Stognienko, R., Henning, T., \& Ossenkopf, V. 1995, A\&A, 296, 797

Su, K. Y. L., Rieke, G. H., Misselt, K. A., et al. 2005, ApJ, 628, 487

Thébault, P. 2012, A\&A, 537, A65

Thébault, P., \& Augereau, J.-C. 2007, A\&A, 472, 169

Thébault, P., \& Wu, Y. 2008, A\&A, 481, 713

Thompson, M. A., Smith, D. J. B., Stevens, J. A., et al. 2010, A\&A, 518, L134 van Leeuwen, F. 2007, A\&A, 474, 653

Vicente, S. M., \& Alves, J. 2005, A\&A, 441, 195

Vilenius, E., Kiss, C., Mommert, M., et al. 2012, A\&A, 541, A94

Vitense, C., Krivov, A. V., \& Löhne, T. 2010, A\&A, 520, A32

Voges, W., Aschenbach, B., Boller, T., et al. 2000, yCat, 9029, 0

Warren, S. G. 1984, ApOpt, 23, 1206

Weidenschilling, S. J. 1980, Icar, 44, 172

White, G. J., Liseau, R., Men'shchikov, A. B., et al. 2000, A\&A, 364, 741

Williams, J. P., \& Cieza, L. A. 2011, ARA\&A, 49, 67

Wilner, D. J., D'Alessio, P., Calvet, N., Claussen, M. J., \& Hartmann, L. 2005, ApJ, 626, L109

Windmark, F., Birnstiel, T., Güttler, C., et al. 2012, A\&A, 540, A73

Wyatt, M. C. 2005, A\&A, 433, 1007

Wyatt, M. C. 2008, ARA\&A, 46, 339

Wyatt, M. C., Clarke, C. J., \& Booth, M. 2011, CeMDA, 111,

Wyatt, M. C., \& Dent, W. R. F. 2002, MNRAS, 334, 589

Wyatt, M. C., Dermott, S. F., Telesco, C. M., et al. 1999, ApJ, 527, 918

Wyatt, M. C., Kennedy, G., Sibthorpe, B., et al. 2012, MNRAS, 424, 1206

Zsom, A., Ormel, C. W., Dullemond, C. P., \& Henning, T. 2011, A\&A, 534, A73

Zsom, A., Ormel, C. W., Güttler, C., Blum, J., \& Dullemond, C. P. 2010, A\&A, 513, A57

Zubko, V. G., Mennella, V., Colangeli, L., \& Bussoletti, E. 1996, MNRAS, 282,1321 